\title{
Domain walls and vortices in linearly coupled systems
}

\author{
Nir Dror, Boris A. Malomed, and Jianhua Zeng
}

We investigate one-dimensional (1D) and 2D radial domain-wall (DW) states in the system of two nonlinear-Schrödinger/Gross-Pitaevskii (NLS/GP) equations, which are coupled by the linear mixing and by the nonlinear XPM (cross-phase-modulation). The system has straightforward applications to two-component Bose-Einstein condensates, and to the bimodal light propagation in nonlinear optics. In the former case, the two components represent different hyperfine atomic states, while in the latter setting they correspond to orthogonal polarizations of light. Conditions guaranteeing the stability of flat continuous wave (CW) asymmetric bimodal states are established, followed by the study of families of the corresponding DW patterns. Approximate analytical solutions for the DWs are found near the point of the symmetry-breaking bifurcation of the CW states. An exact DW solution is produced for ratio $3: 1$ of the XPM and SPM coefficients. The DWs between flat asymmetric states, which are mirror images to each other, are completely stable, and all other species of the DWs, with zero crossings in one or two components, are fully unstable. Interactions between two DWs are considered too, and an effective potential accounting for the attraction between them is derived analytically. Direct simulations demonstrate merger and annihilation of the interacting DWs. The analysis is extended for the system including single- and double-peak external potentials. Generic solutions for trapped DWs are obtained in a numerical form, and their stability is investigated. An exact stable solution is found for the DW trapped by a single-peak potential. In the $2 \mathrm{D}$ geometry, stable two-component vortices are found, with topological charges $s=1,2,3$. Radial oscillations of annular DW-shaped pulsons, with $s=0,1,2$, are studied too. A linear relation between the period of the oscillations and the mean radius of the DW ring is derived analytically.

PACS numbers: 42.65.Tg; 03.75.Lm; 05.45.Yv; 47.20.Ky 


\section{INTRODUCTION}

A ubiquitous type of topologically protected patterns in binary (two-component) nonlinear systems is represented by domain walls (DWs), alias "grain boundaries". A commonly known origin of DWs is in the theory of media with a vectorial local order parameter, such as magnetics [1, ferroelectrics [2], and liquid crystals [3]. In systems described by binary wave functions (at the fundamental or phenomenological level), the DW represents a transient layer between semi-infinite domains carrying different components, or distinct combinations of the components.

A simple but physically significant example is a rectilinear border between two regions occupied by spontaneously emerging roll structures with different orientations. This is, for instance, a generic defect observed in patterns on the surface of thermal-convection layers [4 6]. The theoretical description of such patterns is based on systems of coupled Ginzburg-Landau equations, each equation governing a slowly varying amplitude of a plane wave whose superposition forms the DW [7. Formally similar systems of coupled nonlinear-Schrödinger (NLS) equations and Gross-Pitaevskii (GP) equations describe, respectively, the co-propagation of electromagnetic waves with orthogonal polarizations in nonlinear optical fibers [8, and binary mixtures of Bose-Einstein condensates (BECs) in cigar-shaped traps 9]. While the Ginzburg-Landau systems are dissipative ones, on the contrary to the conservative NLS/GP systems, their stationary versions essentially coincide, hence DW patterns, generated by the stationary equations, are ubiquitous, playing a fundamental role in sundry physical media, both dissipative and conservative ones. Of course, the dynamics of perturbed DW structures may be different in the dissipative and conservative systems.

The basic models which give rise to DWs formed by two fields feature nonlinear XPM (cross-phase-modulation) interaction between the fields. In this context, one-dimensional (1D) solutions for DWs were reported in Ref. [7 for grain boundaries in thermal-convection patterns, in Refs. [10, 11] for temporal-domain DWs between electromagnetic waves with orthogonal circular polarizations in bimodal optical fibers, and (in a number of different forms) in twocomponent BECs [12. Various extensions of these settings were investigated too, including grain boundaries between domains filled by different cellular [13] and quasi-periodic [14 patterns, and between traveling-wave domains in the model of the oscillatory thermal convection [15. Optical DWs between polarized waves with different wavelengths were also studied [16]. Moreover, the analysis was performed for DW states in the discrete version of the nonlinearlycoupled NLS system, which describes arrays of parallel bimodal optical waveguides [17 or BEC fragmented in a deep optical-lattice potential (in the latter case, the system takes the form of the Bose-Hubbard model) [18. Also considered were the transition to immiscibility in binary BEC with long-range dipole-dipole interactions [19], and various forms of DWs in the three-component spinor BEC [20].

As concerns the experiment, linear grain boundaries between patches filled by rolls with different orientations had been well documented in many observations of the thermal convection [5, 21]. Similar linear defects were reported in laser cavities [22] and in experimental studies of BEC [25]. Well-pronounced DW structures have also been created in bimodal optical fibers [23] and in fiber lasers [24].

In many physically relevant settings, the nonlinear interaction between coexisting waves competes with the linear interconversion between them, which strongly affects the DW patterns in such binary systems. In particular, the linear coupling between the orthogonal polarizations induced by the twist or elliptic deformation of the optical fiber gives rise to an effective force accelerating the corresponding DW 11. The linear interconversion between two components of a binary BEC representing different atomic hyperfine states, coupled by a resonant radio-frequency wave, affects the formation of the DW in immiscible binary condensates [26. The objective of the present work is to develop a comprehensive study of DW states in systems of NLS/GP equations coupled by both the linear and nonlinear (XPM) terms, in 1D and 2D geometries. Related two-component vortical states in 2D are studied too.

The paper is organized as follows. The model is formulated in Section II. In Section III, we consider flat continuouswave (CW) states, which represent backgrounds supporting DWs. Both symmetric and asymmetric CW states are found, and the symmetry-breaking bifurcation (SBB), which gives rise to asymmetric CW backgrounds, is identified. The stability of the flat states is also investigated in this section. Basic types of DWs in the free space (without an external potential) are considered in Section IV] where both analytical and numerical solutions for the DWs are reported, and the stability of the DW patterns is established. Section $V$ deals with one and two DWs interacting with an external potential, in the form of one or two peaks. In particular, an analytical stable solution is produced for a DW pinned to a potential peak. Two-dimensional axisymmetric patterns are considered in Section VI. Stable vortices with topological charges $s=1,2,3$, supported by the asymmetric CW background, are found, and radial oscillations of annular pulsons in the form of circular DWs are studied too. The paper is concluded by Section VII. 


\section{THE MODEL}

Our starting point is the system of scaled one-dimensional NLS/GP equations for two wave functions $\psi_{1,2}$, coupled by the linear and nonlinear (XPM) terms:

$$
\begin{aligned}
& i\left(\psi_{1}\right)_{t}=-(1 / 2)\left(\psi_{1}\right)_{x x}+\sigma\left|\psi_{1}\right|^{2} \psi_{1}+g\left|\psi_{2}\right|^{2} \psi_{1}-\kappa \psi_{2}, \\
& i\left(\psi_{2}\right)_{t}=-(1 / 2)\left(\psi_{2}\right)_{x x}+\sigma\left|\psi_{2}\right|^{2} \psi_{2}+g\left|\psi_{1}\right|^{2} \psi_{2}-\kappa \psi_{1},
\end{aligned}
$$

where $\kappa$ is the rate of the linear interconversion between the two atomic states, if the system is interpreted in terms of BEC 26. The same equations, with time $t$ replaced by the propagation distance, $z$, and $x$ replaced by the reduced time, $\tau \equiv t-z / V_{\mathrm{gr}}$, where $V_{\mathrm{gr}}$ is the group velocity of the carrier wave, may be realized in optics as the model of the light propagation in an ordinary or photonic-crystal fiber [8]. In the fiber-optic model, amplitudes $\psi_{1,2}$ represent two mutually orthogonal polarizations of light, with the linear interconversion induced by the birefringence or twist of the fiber, for the circular or linear polarizations, respectively. Coefficients $\sigma$ and $g$ in Eqs. (1) account for the SPM (self-phase-modulation) and XPM nonlinearities, respectively (in the ordinary optical fiber, the XPM/SPM ratio is $g / \sigma=2$ and $4 / 3$ for the of circular and linear polarizations, respectively).

To secure the modulational stability of CW states supporting DWs, coefficient $g$ will be kept positive, which corresponds to the repulsive XPM nonlinearity, while SPM coefficient $\sigma$ may have any sign. In optics, opposite signs of the XPM and SPM coefficients is an exotic situation, which is, nevertheless, possible in photonic-crystal fibers [8]. In BEC, the sign of either coefficient may be switched by means of the Feshbach-resonance effect 9 .

The value of $g$ may be fixed by dint of an obvious rescaling [for instance, it is possible to set $g \equiv 3$, which is a natural choice in view of the existence of an exact DW solution in the form of Eqs. (36) and (37), see below, which requires $g=3 \sigma]$. In addition, the rescaling allows one to fix $|\kappa| \equiv 1$, thus we will assume $\kappa= \pm 1$. Nevertheless, in the analytical expressions written below, we keep $g$ and $\kappa$ as free parameters, as it is easier to analyze the results in such a form.

Stationary solutions to Eqs. (1) with chemical potential $\mu$ are sought for as

$$
\psi_{1,2}(x, t)=e^{-i \mu t} \phi_{1,2}(x)
$$

with real functions $\phi_{1,2}(x)$ satisfying equations

$$
\begin{aligned}
& \mu \phi_{1}+(1 / 2) \phi_{1}^{\prime \prime}-\sigma \phi_{1}^{3}-g \phi_{2}^{2} \phi_{1}+\kappa \phi_{2}=0 \\
& \mu \phi_{2}+(1 / 2) \phi_{2}^{\prime \prime}-\sigma \phi_{2}^{3}-g \phi_{1}^{2} \phi_{2}+\kappa \phi_{1}=0
\end{aligned}
$$

with the prime standing for $d / d x$. The energy (Hamiltonian) corresponding to stationary states $(2)$ is $H=\int_{-\infty}^{+\infty} \mathcal{H} d x$, with density

$$
\begin{gathered}
\mathcal{H}=(1 / 2)\left[\left(\phi_{1}^{\prime}\right)^{2}+\left(\phi_{2}^{\prime}\right)^{2}\right] \\
+(\sigma / 2)\left(\phi_{1}^{4}+\phi_{2}^{4}\right)+g \phi_{1}^{2} \phi_{2}^{2}-2 \kappa \phi_{1} \phi_{2} .
\end{gathered}
$$

\section{FLAT (CONTINUOUS-WAVE) STATES}

Symmetric flat ( $x$-independent) solutions to Eqs. (3), with equal amplitudes of both components, are

$$
\phi_{1}=\phi_{2} \equiv A_{0}=\sqrt{(\mu+\kappa) /(\sigma+g)} \text {. }
$$

The Hamiltonian density (4) for this solution is

$$
\mathcal{H}_{\mathrm{symm}}=\left(\mu^{2}-\kappa^{2}\right) /(g+\sigma)
$$

Antisymmetric CW solutions are equivalent to Eq. 5 with $\kappa$ replaced by $-\kappa$. In view of this relation, we will define the symmetric solutions as those for $\kappa=+1$, while the antisymmetric ones will be replaced by symmetric states for $\kappa=-1$. 
Asymmetric flat states, which are generated by the SBB, can also be found in the exact form:

$$
\begin{aligned}
& \phi_{1}^{2}=\frac{\mu}{2 \sigma} \pm \sqrt{\frac{\mu^{2}}{4 \sigma^{2}}-\frac{\kappa^{2}}{(g-\sigma)^{2}}} \equiv A_{1}^{2}, \\
& \phi_{2}^{2}=\frac{\mu}{2 \sigma} \mp \sqrt{\frac{\mu^{2}}{4 \sigma^{2}}-\frac{\kappa^{2}}{(g-\sigma)^{2}}} \equiv A_{2}^{2},
\end{aligned}
$$

with the signs of $\phi_{1}$ and $\phi_{2}$ determined by relation

$$
\phi_{1} \phi_{2}=\kappa(g-\sigma)^{-1}
$$

which is compatible with Eqs. (7). The Hamiltonian density (4) for these states is

$$
\mathcal{H}_{\text {asymm }}=\mu^{2} /(2 \sigma)-\kappa^{2} /(g-\sigma) .
$$

Taking into account the above convention, $\phi_{1} \phi_{2}>0$, and the condition that $\phi_{1,2}^{2}$ must be positive, it follows from Eqs. (7) that the asymmetric CW state emerges, i.e., the SBB takes place, at a specific value of the chemical potential,

$$
\mu=2 \sigma \kappa(g-\sigma)^{-1} \equiv \mu_{\mathrm{cr}},
$$

the corresponding value of $A_{0}^{2}$ at the bifurcation point being obtained by the substitution of this value into Eq. (5):

$$
A_{\mathrm{cr}}^{2} \equiv \kappa(g-\sigma)^{-1} \text {. }
$$

The asymmetric solution (8), (7) exists at $\mu^{2}>\mu_{\mathrm{cr}}^{2}$. Slightly above the bifurcation point, i.e., at

$$
\mu=\mu_{\mathrm{cr}}+\delta \mu, \text { with }|\delta \mu| \ll\left|\mu_{\mathrm{cr}}\right|,
$$

one can expand Eqs. (7) and (8) in powers of small $\delta \mu$, which yields

$$
\phi_{1,2}=\sqrt{\frac{\kappa}{g-\sigma}} \pm \frac{1}{2} \sqrt{\frac{\delta \mu}{\sigma}}+\mathcal{O}(\delta \mu) .
$$

\section{A. The modulational stability of the symmetric solution at the bifurcation point}

A crucial condition necessary for the existence of stable DWs is the absence of the modulational instability (MI) of the corresponding CW background. Here we explicitly consider the MI of the symmetric CW, and, in particular, we will find conditions providing for the stability of the symmetric state exactly at the SBB point. Examining this case secures that the asymmetric CW states are not subject to the MI - at least, close enough to the bifurcation point.

To analyze the MI, we look for perturbed solutions to Eqs. (1) in the well-known general form, i.e., as

$$
\left.\psi_{1,2}(x, t)=\left[A_{0}+a_{1,2}(x, t)\right] \exp \left[-i \mu t+i \chi_{1,2}(x, t)\right)\right]
$$

where amplitude $A_{0}$ is the same as in Eq. (5), while $a_{1,2}$ and $\chi_{1,2}$ are infinitesimal perturbations of the amplitudes and phases of the two components (this form of the solution implies the incorporation of the small complex perturbations into the unperturbed CW state). Then, eigenmodes of the perturbations are sought for as

$$
\left\{a_{1,2}(x, t), \chi_{1,2}(x, t)\right\}=\left\{a_{1,2}^{(0)}, \chi_{1,2}^{(0)}\right\} \exp (\gamma t+i p x),
$$

where $p$ is an arbitrary real wavenumber of the perturbation, and $\gamma$ the corresponding instability gain, which may be complex. The stability condition is that $\operatorname{Re}\{\gamma(p)\} \equiv 0$ for all real $p$.

The substitution of expressions (14) and (15) into Eqs. (1) and linearization with respect to infinitesimal perturbations yields the following system of equations, which actually splits into two separate subsystems for $\left(a_{1}^{(0)}+a_{2}^{(0)}\right)$, $\left(\chi_{1}^{(0)}+\chi_{2}^{(0)}\right)$ and $\left(a_{1}^{(0)}-a_{2}^{(0)}\right),\left(\chi_{1}^{(0)}-\chi_{2}^{(0)}\right)$ :

$$
\begin{array}{r}
\gamma\left(a_{1}^{(0)}+a_{2}^{(0)}\right)-(1 / 2) A_{0} p^{2}\left(\chi_{1}^{(0)}+\chi_{2}^{(0)}\right)=0 \\
{\left[2 A^{2}(g+\sigma)+(1 / 2) p^{2}\right]\left(a_{1}^{(0)}+a_{2}^{(0)}\right)+\gamma A_{0}\left(\chi_{1}^{(0)}+\chi_{2}^{(0)}\right)=0}
\end{array}
$$




$$
\begin{array}{r}
\gamma\left(a_{1}^{(0)}-a_{2}^{(0)}\right)-\left[(1 / 2) p^{2}+2 \kappa\right] A_{0}\left(\chi_{1}^{(0)}-\chi_{2}^{(0)}\right)=0, \\
{\left[2 A^{2}(g-\sigma)-2 \kappa-(1 / 2) p^{2}\right]\left(a_{1}^{(0)}-a_{2}^{(0)}\right)-\gamma A_{0}\left(\chi_{1}^{(0)}-\chi_{2}^{(0)}\right)=0 .}
\end{array}
$$

The resolvability conditions for Eqs. (16) and (17) yield, respectively, the following expressions for $\gamma(p)$ :

$$
\begin{aligned}
& \gamma_{+}^{2}=-(1 / 2) p^{2}\left[2(g+\sigma) A_{0}^{2}+(1 / 2) p^{2}\right] \\
& \gamma_{-}^{2}=\left(2 \kappa+(1 / 2) p^{2}\right)\left[2(g-\sigma) A_{0}^{2}-2 \kappa-(1 / 2) p^{2}\right] .
\end{aligned}
$$

The stability condition ensuing from Eq. 18 , i.e., $\gamma_{+}^{2}<0$, is obvious:

$$
g+\sigma>0 \text {. }
$$

Expression 19 simplifies at the bifurcation point 10 , where $A_{0}^{2}=\kappa /(g-\sigma)$, as per Eq. (11):

$$
\gamma_{-}^{2}=-(1 / 2) p^{2}\left[2 \kappa+(1 / 2) p^{2}\right]
$$

Evidently, the stability condition following from Eq. 21 is $\kappa>0$, which, as a matter of fact, means that only the symmetric flat solution may be stable at the SBB point, while its antisymmetric counterpart, that (as defined above) corresponds to $\kappa<0$, is unstable. Further, from $\kappa>0$ and the positiveness of expression (11) for $A_{\mathrm{cr}}^{2}$, condition $g-\sigma>0$ follows. Combined with Eq. (20), it gives rise to the following relation between the XPM and SPM coefficients necessary for the existence and stability of the asymmetric CW states:

$$
|\sigma|<g
$$

while $\sigma$ may be positive or negative. In fact, it will be demonstrated below that DW solutions do not exists for $\sigma<0$.

Note that condition (22) does not hold for $g=0$, hence the DWs that can be found in the system with the solely linear coupling are always unstable, as demonstrated in Ref. 27. In that work, it was shown that the unstable DW gives rise to an expanding layer filled with turbulent waves.

\section{B. The modulational stability of the asymmetric background}

In the general case of the asymmetric CW background, the perturbed solution is taken as [cf. Eq. (14)]

$$
\left.\psi_{1,2}(x, t)=A_{1,2}\left[1+b_{1,2}(x, t)\right] \exp \left[-i \mu t+i \chi_{1,2}(x, t)\right)\right],
$$

where $A_{1,2}$ are given by Eqs. (7), and infinitesimal perturbations are taken as [cf. Eqs. [15]

$$
\left\{b_{1,2}(x, t), \chi_{1,2}(x, t)\right\}=\left\{b_{1,2}^{(0)}, \chi_{1,2}^{(0)}\right\} \exp (\gamma t+i p x) .
$$

The substitution of expressions (23) and (24) into Eqs. (1), and the subsequent linearization, lead to a system of four linear equations:

$$
\begin{aligned}
\gamma b_{1}^{(0)}-\frac{1}{2} p^{2} \chi_{1}^{(0)}-\kappa \frac{A_{2}}{A_{1}}\left(\chi_{1}^{(0)}-\chi_{2}^{(0)}\right) & =0, \\
\gamma b_{2}^{(0)}-\frac{1}{2} p^{2} \chi_{2}^{(0)}+\kappa \frac{A_{1}}{A_{2}}\left(\chi_{1}^{(0)}-\chi_{2}^{(0)}\right) & =0, \\
\gamma \chi_{1}^{(0)}+\frac{1}{2} p^{2} b_{1}^{(0)}+2 \sigma A_{1}^{2} b_{1}^{(0)}+2 g A_{2}^{2} b_{2}^{(0)}+\kappa \frac{A_{2}}{A_{1}}\left(b_{1}^{(0)}-b_{2}^{(0)}\right) & =0, \\
\gamma \chi_{2}^{(0)}+\frac{1}{2} p^{2} b_{2}^{(0)}+2 \sigma A_{2}^{2} b_{2}^{(0)}+2 g A_{1}^{2} b_{1}^{(0)}-\kappa \frac{A_{1}}{A_{2}}\left(b_{1}^{(0)}-b_{2}^{(0)}\right) & =0 .
\end{aligned}
$$

In the special case of $A_{1}=A_{2} \equiv A_{0}$, it is easy to check that Eqs. 25) are tantamount to Eqs. (16) and (17) written above.

The dispersion relation between $\gamma$ and $p^{2}$ is determined by the resolvability condition of system 25):

$$
\left|\begin{array}{cccc}
\gamma & 0 & -\left(\kappa \frac{\phi_{2}}{\phi_{1}}+\frac{1}{2} p^{2}\right) & \kappa \frac{\phi_{2}}{\phi_{1}} \\
0 & \gamma & \kappa \frac{\phi_{1}}{\phi_{2}} & -\left(\kappa \frac{\phi_{1}}{\phi_{2}}+\frac{1}{2} p^{2}\right) \\
2 \sigma \phi_{1}^{2}+\kappa \frac{\phi_{2}}{\phi_{1}}+\frac{1}{2} p^{2} & 2 g \phi_{2}^{2}-\kappa \frac{\phi_{2}}{\phi_{1}} & \gamma & 0 \\
2 g \phi_{1}^{2}-\kappa \frac{\phi_{1}}{\phi_{2}} & 2 \sigma \phi_{2}+\kappa \frac{\phi_{1}}{\phi_{2}}+\frac{1}{2} p^{2} & 0 & \gamma
\end{array}\right|=0 .
$$


Equation (26) was solved numerically, yielding four roots $\gamma\left(p^{2}\right)$. It has been verified that, with $A_{1}$ and $A_{2}$ taken as per Eqs. (7) and (8), and for all $p^{2} \geq 0$, all the roots satisfy condition $\operatorname{Re}\left\{\gamma\left(p^{2}\right)\right\}=0$, if $\kappa$ is positive and inequality 22 holds. Thus, as long as the symmetric CW are stable at the SBB point, its asymmetric counterparts are stable too.

\section{DOMAIN-WALL SOLUTIONS IN THE FREE SPACE}

\section{A. Numerical results}

Stationary solutions to equations (3) were constructed by means of the Newton-Raphson method for the corresponding nonlinear boundary-value problem. In particular, the boundary conditions were fixed as zero values of the derivatives at both edges of the integration domain.

DWs are built as transient layers fusing together CW states of different types. Obviously, such patterns are possible at $\mu^{2}>\mu_{\mathrm{cr}}^{2}$ [see Eq. [10]], where asymmetric CW solutions exist, as given by Eqs. (7). Figure 1] shows that, in this case, one can find four different types of the transient layers, if dark solitons are counted too. These types may be classified by values of coupled fields $\left(\phi_{1}, \phi_{2}\right)$ in the uniform states connected by the DWs:

$$
\begin{aligned}
& \left\{\left(\phi_{1}(x=-\infty), \phi_{2}(x=-\infty)\right),\left(\phi_{1}(x=+\infty), \phi_{2}(x=+\infty)\right)\right\} \\
& =\left\{\left(A_{1}, A_{2}\right),\left(A_{2}, A_{1}\right)\right\} ;\left\{\left(A_{1}, A_{2}\right),\left(-A_{2},-A_{1}\right)\right\} \\
& \left\{\left(A_{1}, A_{2}\right),\left(-A_{1},-A_{2}\right)\right\} ;\left\{\left(A_{0}, A_{0}\right),\left(-A_{0},-A_{0}\right)\right\}
\end{aligned}
$$

[recall that $A_{0}$ is given by Eq. (5), and $A_{1,2}$ are given by Eqs. (7)]. In fact, only the patterns of the first and second types in Eq. (27) [Figs. 1(a) and (b)] are true DWs, while Figs. 1(c) and (d), corresponding to the profiles of the third and fourth types in Eq. (27), are paired dark solitons. It is also clear that the dark-soliton pairs of the latter type, displayed in Fig. 1(d), are unstable past the SBB point, as symmetric CW state (5) is unstable in this case.

It is relevant to mention that DWs connecting the symmetric and asymmetric states - for instance, $\left(A_{1}, A_{2}\right)$ and $\left(A_{0}, A_{0}\right)$ - are impossible, because a stationary DW may only exist between two asymptotic flat states with equal Hamiltonian densities [11. Comparing the respective densities (6) and (9), one can immediately conclude that they coincide solely at the bifurcation point 10 .

To investigate the stability of the DW patterns, small perturbations were added to the stationary solutions:

$$
\begin{aligned}
& \widetilde{\phi_{1}}(x, t)=\phi_{1}(x)+v_{1}(x) e^{-i \gamma t}+u_{1}^{*}(x) e^{i \gamma^{*} t}, \\
& \widetilde{\phi_{2}}(x, t)=\phi_{2}(x)+v_{2}(x) e^{-i \gamma t}+u_{2}^{*}(x) e^{i \gamma^{*} t},
\end{aligned}
$$

where $v_{1}, u_{1}$ and $v_{2}, u_{2}$ constitute eigenmodes of the infinitesimal perturbation, and $\gamma$ is the corresponding eigenfrequency that, in general, may be complex. Substituting expressions (28) into Eqs. (3) and linearizing around the stationary solutions leads to the following eigenvalue problem,

$$
\begin{gathered}
\left(\begin{array}{cccc}
-\hat{L_{1}} & \sigma \phi_{1}^{2} & g \phi_{1} \phi_{2}-\kappa & g \phi_{1} \phi_{2} \\
-\sigma \phi_{1}^{2} & \hat{L_{1}} & -g \phi_{1} \phi_{2} & -g \phi_{1} \phi_{2}+\kappa \\
g \phi_{2} \phi_{1}-\kappa & g \phi_{2} \phi_{1} & -\hat{L_{2}} & \sigma \phi_{2}^{2} \\
-g \phi_{2} \phi_{1} & -g \phi_{2} \phi_{1}+\kappa & -\sigma \phi_{2}^{2} & \hat{L}_{2}
\end{array}\right)\left(\begin{array}{c}
v_{1} \\
u_{1} \\
v_{2} \\
u_{2}
\end{array}\right)=\gamma\left(\begin{array}{c}
v_{1} \\
u_{1} \\
v_{2} \\
u_{2}
\end{array}\right), \\
\hat{L_{1}} \equiv \mu+(1 / 2) d^{2} / d x^{2}-2 \sigma \phi_{1}^{2}-g \phi_{2}^{2} \\
\hat{L_{2}} \equiv \mu+(1 / 2) d^{2} / d x^{2}-2 \sigma \phi_{2}^{2}-g \phi_{1}^{2}
\end{gathered}
$$

This eigenvalue problem can be solved using a simple finite-difference scheme. Accordingly, the solution is identified as a stable one if all the eigenfrequencies are real.

The stability analysis outlined above demonstrates that the DW family of the first type in Eq. (27), which is represented by Fig. $1(\mathrm{a})$ is completely stable (past the SBB point, where it exists, along with the asymmetric CW states). In this case, all the other types of the DW and dark-soliton solutions [i.e., all of them which cross zero at least in one component, see Fig. 1 (b,c,d)] are completely unstable, due to the presence of imaginary eigenfrequencies in the spectrum of small perturbations. We stress that, unlike the trivial background instability of the pattern of the last type in Eq. 27), those of the second and third types, which are represented by Figs. 1(b) and (c) are destabilized by perturbations localized around the transient layer, while the $\mathrm{CW}$ background is stable. Direct simulations demonstrate that these unstable DWs decay into expanding turbulent patterns (see Fig. 22). The stability of the DW of the first type in Eq. (27) was also verified by direct simulations (not shown here). 

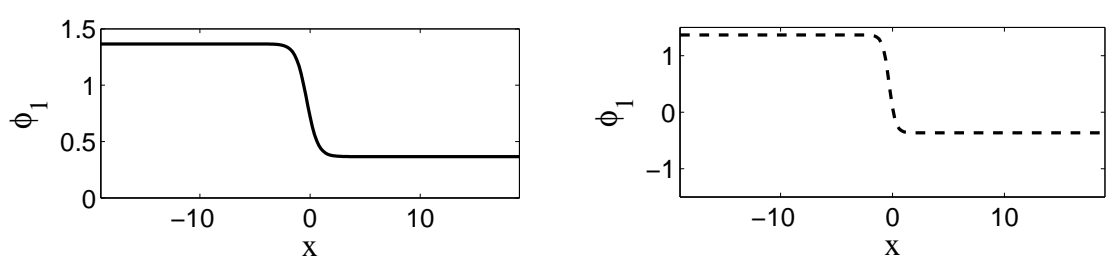

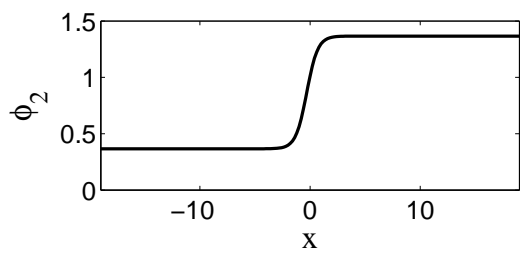

(a)
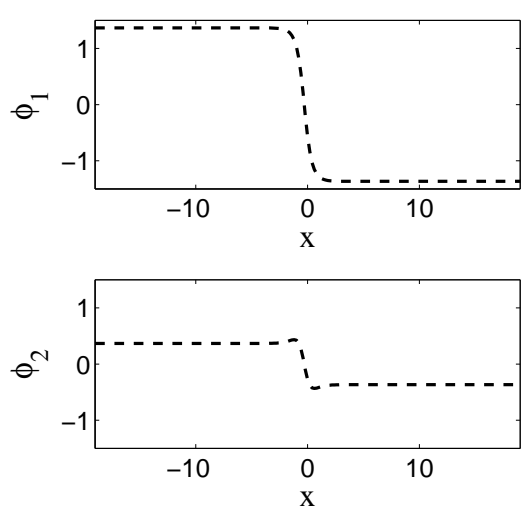

(c)

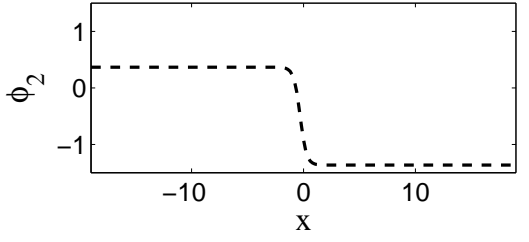

(b)
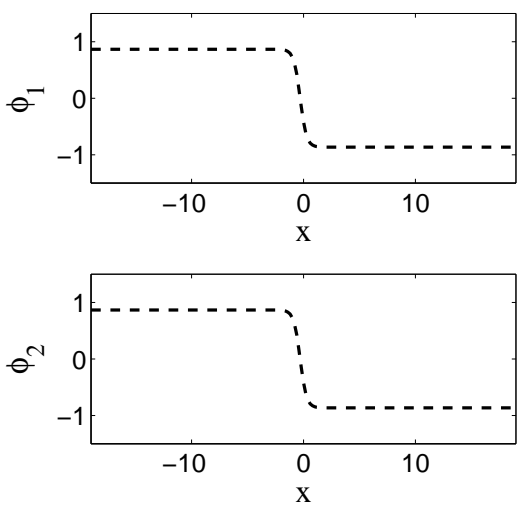

(d)

FIG. 1. Typical examples of the domain walls (a, b) and paired dark solitons (c, d), found for $g=3, \sigma=\kappa=1$, and $\mu=2$. The four panels represent the patterns of the four types defined in Eq. (27). Stable and unstable solutions are depicted by the continues and dashed lines, respectively.

\section{B. An approximate solution for the DW near the bifurcation point}

An analytical solution for the DW can be constructed in an approximate asymptotic form near the bifurcation point (10), 111), i.e., for $\mu$ taken as in Eq. (12). In this case, the approximate solution can be sought for as

$$
\begin{aligned}
& \phi_{1}(x)=A_{\mathrm{cr}}+\delta \phi_{1}(x)+\delta \phi_{2}(x), \\
& \phi_{2}(x)=A_{\mathrm{cr}}-\delta \phi_{1}(x)+\delta \phi_{2}(x),
\end{aligned}
$$

where it is implied that $\delta \phi_{1} \sim \sqrt{\delta \mu}$ and $\delta \phi_{2} \sim \delta \mu$, cf. Eq. (13). Substituting expressions (31) into Eqs. (3) and expanding the result in powers of $\delta \mu$ yields a relation between $\delta \phi_{1}$ and $\delta \phi_{2}$ at order $\delta \mu$,

$$
\delta \phi_{2}(x)=\frac{A_{\mathrm{cr}}}{2(\mu+\kappa)}\left[\delta \mu+(g-3 \sigma)\left(\delta \phi_{1}(x)\right)^{2}\right] .
$$

Next, at order $\delta \mu^{3 / 2}$ the expansion yields the equation for $\delta \phi_{1}(x)$ :

$$
\left(\delta \phi_{1}\right)^{\prime \prime}+4 \frac{g-\sigma}{g+\sigma} \delta \mu \cdot \delta \phi_{1}-16 \sigma \frac{g-\sigma}{g+\sigma}\left(\delta \phi_{1}\right)^{3}=0 .
$$

An exact solution of the DW type to Eq. (33) is

$$
\delta \phi_{1}(x)=\frac{1}{2} \sqrt{\frac{\delta \mu}{\sigma}} \tanh \left(\sqrt{2 \frac{g-\sigma}{g+\sigma} \delta \mu x}\right) .
$$



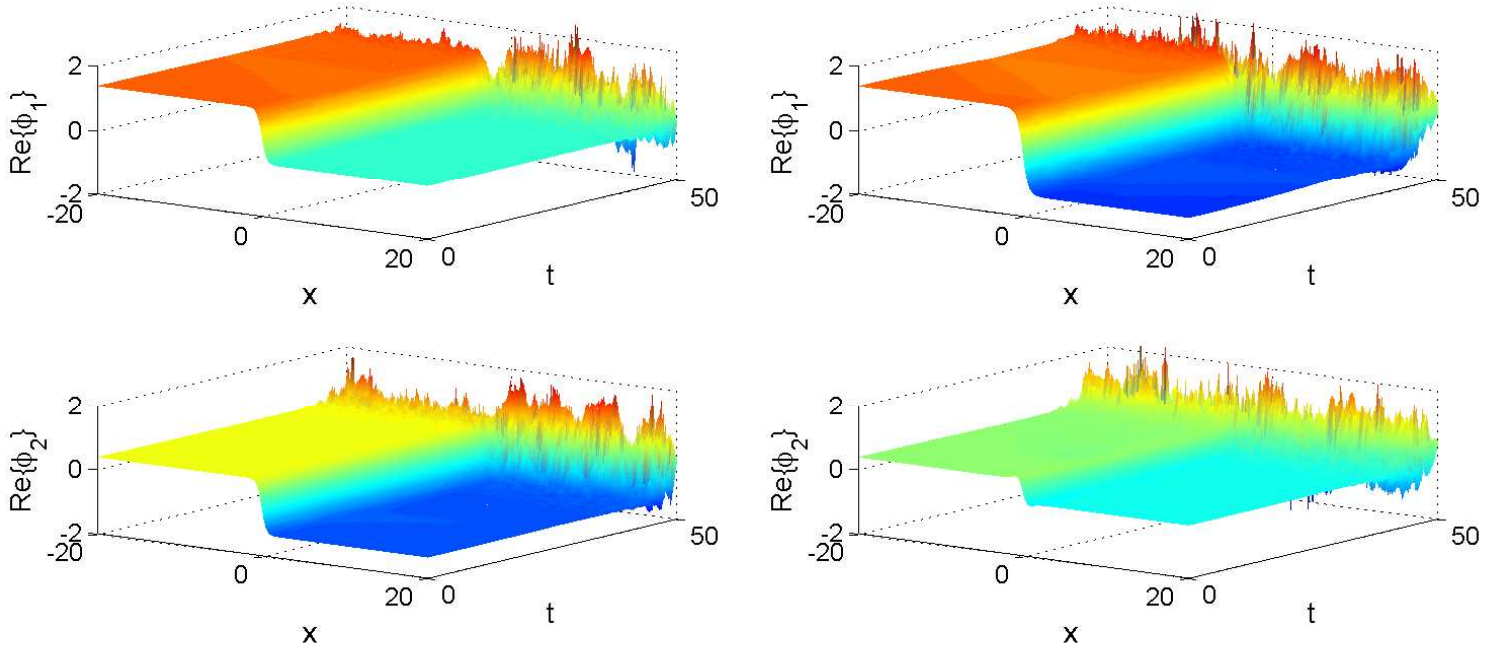

(a)

(b)

FIG. 2. (Color online) Examples for the evolution of unstable patterns of the second (a) and third (b) types from Eq. 27). The initial conditions and the parameters are as in Fig. 1(b) and (c) for (a) and (b), respectively.

With regard to the CW-stability condition (22), we conclude that solution (34) exists if the sign of $\delta \mu$ coincides with the sign of $\sigma$, and only for $\delta \mu>0$, which means that the solution exists solely for $\sigma>0$. In fact, the numerical solution demonstrates too that DWs cannot be found at $\sigma<0$, even if asymmetric CW states (7) exist in this case, with $\mu<0$.

In what follows below, we will also need an expression for the full density of the DW solution. The above formulas yield

$$
\begin{aligned}
& {\left[\phi_{1}(x)\right]^{2}+\left[\phi_{2}(x)\right]^{2} } \\
= & \frac{2 \kappa}{g-\sigma}+\frac{\delta \mu}{\sigma}-\frac{g-\sigma}{g+\sigma} \frac{\delta \mu}{\sigma} \operatorname{sech}^{2}\left(2 \sqrt{\frac{g-\sigma}{g+\sigma} \delta \mu x}\right) .
\end{aligned}
$$

Examples of the analytically predicted profiles, as given by Eqs. (31), (32) and (34), together with their numerically found counterparts, are displayed in Fig. $3(\mathrm{a})$, for parameters $g=3, \sigma=1, \kappa=1$ [i.e., $\mu_{\text {cr }}=1$, see Eq. (10)] and $\delta \mu=0.1,0.5,1$ and 2. For the same examples, the difference between the numerical and approximate results is shown in Fig. 3(b) The results presented in Fig. 3(a) demonstrate that the prediction loses its accuracy with the increase of $\delta \mu$. On the other hand, comparing the core of the analytical and numerical solutions (the transient layer), one can see that the analytical results are not necessarily most accurate for small $\delta \mu$. Actually, this approximation is most suitable for intermediate values of $\delta \mu$.

\section{The exact solution for $g=3 \sigma$}

A particular exact solution to Eqs. (3) for the DW can be found by means of the following ansatz:

$$
\begin{aligned}
& \phi_{1}(x)=U_{0}+U_{1} \tanh (\lambda x), \\
& \phi_{2}(x)=U_{0}-U_{1} \tanh (\lambda x) .
\end{aligned}
$$

The substitution of the ansatz into Eqs. (3) demonstrates that it yields an exact solution at $g=3 \sigma$, for chemical potential $\mu=\kappa+\lambda^{2}$, with coefficients

$$
\begin{aligned}
\lambda^{2} & =\mu-\kappa, U_{0}^{2}=(4 \sigma)^{-1}\left(2 \kappa+\lambda^{2}\right) \equiv(4 \sigma)^{-1}(\mu+\kappa), \\
U_{1}^{2} & =(4 \sigma)^{-1} \lambda^{2} \equiv(4 \sigma)^{-1}(\mu-\kappa)
\end{aligned}
$$



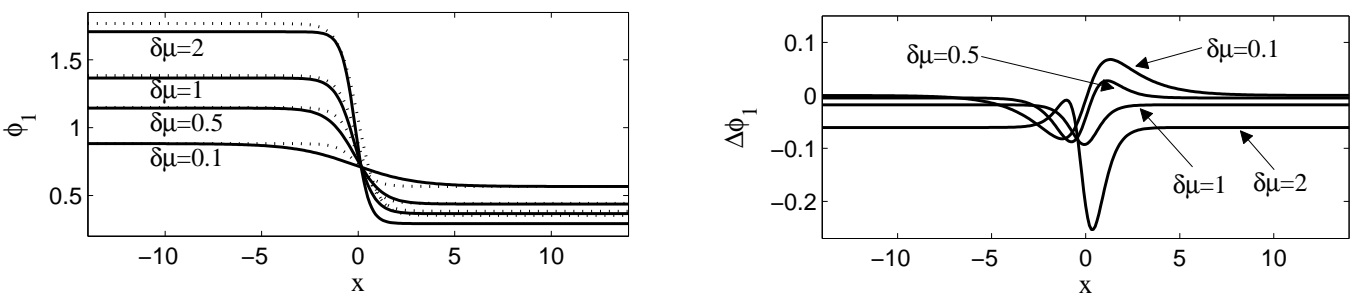

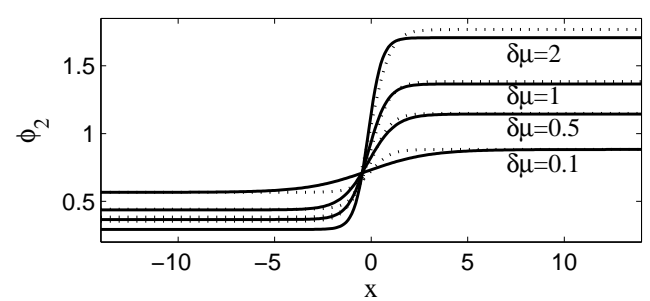

(a)

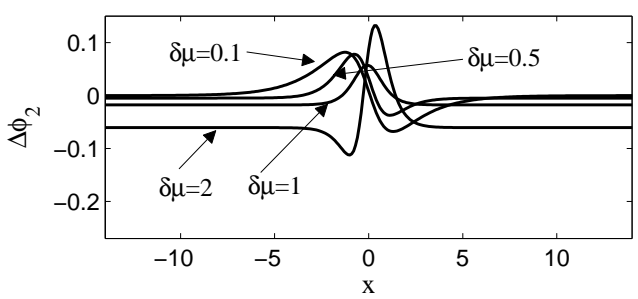

(b)

FIG. 3. (a) Comparison between the analytical approximation given by Eq. (34), which is shown by dotted lines, and numerically found profiles of the domain walls (solid lines), for $g=3, \sigma=1, \kappa=1$ ( $\mu_{\mathrm{cr}}=1$ ) and $\delta \mu=0.1,0.5,1$ and 2 . (b) The differences between the numerical and approximate analytical results, $\Delta \phi_{1,2}=\left(\phi_{1,2}\right)_{\text {numer }}-\left(\phi_{1,2}\right)_{\text {approx }}$, for the examples presented in panel (a).

(under condition $\mu-\kappa>0$ ). In fact, this particular exact stationary solution is similar to the one that was found, also in the exact form, for the stationary version of coupled Ginzburg-Landau equations (but without the linear coupling) in Ref. 7. Note that relation $g=3 \sigma$, which is necessary for the existence of the exact solution, complies with the $\mathrm{CW}$-stability condition (22). In terms of this exact solution, $\mu$ may be considered as a free parameter, i.e., we actually have a one-parameter solution family, under constraint $g=3 \sigma$. An example of the exact DW solution can be seen in Fig. 1(a), where the parameters were chosen to comply with the exact solution. Finally, the total local density of solution (36) is

$$
\phi_{1}^{2}(x)+\phi_{2}^{2}(x)=2\left[\left(U_{0}^{2}+U_{1}^{2}\right)-U_{1}^{2} \operatorname{sech}^{2}(\lambda x)\right],
$$

cf. Eq. (35).

It may also be relevant to mention that, under condition $g=3 \sigma$, Eqs. (3) has another formal DW-like solution, given by Eqs. (36) and (37) with tanh replaced by coth. However, this additional solution is singular, therefore it has no physical meaning.

\section{DOMAIN WALLS IN THE PRESENCE OF EXTERNAL POTENTIALS}

The model based on Eqs. (3) can be naturally extended to include an external potential, $W(x)$ :

$$
\begin{aligned}
& \mu \phi_{1}+(1 / 2) \phi_{1}^{\prime \prime}-\sigma \phi_{1}^{3}-g \phi_{2}^{2} \phi_{1}+\kappa \phi_{2}-W(x) \phi_{1}=0, \\
& \mu \phi_{2}+(1 / 2) \phi_{2}^{\prime \prime}-\sigma \phi_{2}^{3}-g \phi_{1}^{2} \phi_{2}+\kappa \phi_{1}-W(x) \phi_{2}=0 .
\end{aligned}
$$

In this section we report the analysis performed for the DW patterns supported by this setting, with $W(x)$ representing single- or double-peak potentials, with the intention to predict pinning of the DWs by such potentials. Stable pinning by potential maxima (rather than minima) may be possible because the DW's core features a minimum of the total density, see Eqs. (35) and (38), hence the total energy of the system may be minimized by placing the core around a local maximum of the potential. 


\section{A. The exact solution in the presence of a single-peak potential}

It is possible to find an exact solution to Eqs. (39) for the DW if the potential is chosen as

$$
W(x)=W_{0} \operatorname{sech}^{2}(\lambda x)
$$

where $W_{0}$ and $\lambda$ are considered as given parameters. The corresponding solution can be looked for in the form of the same ansatz (36) as used for finding the exact solution in the free-space setting. The substitution of the ansatz into Eqs. (39) demonstrates that it yields an exact solution in the present case under the following condition imposed on parameters of the system:

$$
g=\frac{3 \lambda^{2}+2 W_{0}}{\lambda^{2}+2 W_{0}} \sigma
$$

which goes over into the above relation, $g=3 \sigma$, in the limit of $W_{0}=0$. Further, coefficients $U_{0}, U_{1}$, and $\mu$ of the exact solution are given by the following expressions:

$$
\begin{aligned}
\mu & =\lambda^{-2}\left(\lambda^{2}+2 W_{0}\right)\left(\lambda^{2}+\kappa\right), \\
U_{0}^{2} & =\left(4 \sigma \lambda^{2}\right)^{-1}\left(\lambda^{2}+2 W_{0}\right)\left(\lambda^{2}+2 \kappa\right), \\
U_{1}^{2} & =(4 \sigma)^{-1}\left(\lambda^{2}+2 W_{0}\right) .
\end{aligned}
$$

It is easy to see that, in the limit of $W_{0}=0$, Eqs. 42 carry over into the above exact solution, given by Eqs. (37). Thus, this exact solution is an extension of the previous one, although it has no free parameters [note that $\lambda$, which was an adjustable parameter of the free-space solution, is now fixed by the given form of potential [40]].

As argued above, the present exact solution is expected to be stable if $W(x)$ represents a repulsive potential barrier (peak), with $W_{0}>0$, and the solution should be unstable, against spontaneous escape from the pinned state, in the case of the attractive potential well, with $W_{0}<0$ [cf. Eq. (48) below]. In turn, Eq. (41) with $W_{0}>0$ gives $g<3 \sigma$. Note also that, unlike its counterpart in the free space, this exact solution may exist at $\sigma<0$ : In the case of the potential well $\left(W_{0}<0\right)$, with $\lambda^{2}<-2 W_{0}<3 \lambda^{2}$, Eq. (41) yields $\sigma<0$. However, this solution should be unstable according to the above argument.

\section{B. Analysis of the interaction between DWs and the interaction of the DW with the external potential}

The interaction of the DW with an external potential can be investigated in an approximate form. In fact, a similar problem which also admits an approximate analytical treatment is the interaction between two broadly separated DWs with opposite polarities (i.e., mirror images of each other) in the free space, therefore we start with this case.

Assuming that the two DWs are set at distance $L$ which is large in comparison with the inner width of each DW, the interaction between them can be analyzed by means of the method elaborated in Ref. 28. To this end, the approximate expression for the nearly flat fields in the region between the far separated solitons is taken as

$$
\phi_{n}(x)=A_{n}+2 U_{n}\left[\exp \left(-2 \lambda\left|x-\xi_{1}\right|\right)+\exp \left(-2 \lambda\left|x-\xi_{2}\right|\right)\right],
$$

where $n=1,2$, and $A_{1,2}$ are given by Eqs. (7), $\xi_{1,2}$ are coordinates of the centers of the two DWs, so that $L \equiv \xi_{2}-\xi_{1}$, and the exponential terms represent small decaying tails of the DWs on top of the flat background. The decay rate $\lambda>0$ can be found in the general case, but the expression for it is cumbersome; amplitude $U_{1,2}$ are not known in an exact form in the general case, as they may only be found from full solutions for individual DWs. In the special case of the exact solution given by Eqs. (36), at $g=3 \sigma)$, coefficients $\lambda$ and $U_{1,2}$ in Eq. (43) are actually given by Eq. (37):

$$
\lambda=\sqrt{\mu-\kappa}, U_{1}=U_{2}=(1 / 2) \sqrt{(\mu-\kappa) / \sigma} .
$$

Then, using Hamiltonian produced by density (4) and the method developed in Ref. [28] (identifying the term accounting for the interaction energy in the expression for the full Hamiltonian), the effective potential of the interaction between the two separated DWs is found in the following form:

$$
U_{\text {int }}(L)=-8 \lambda\left(U_{1}^{2}+U_{2}^{2}\right) \exp (-2 \lambda L) .
$$

In the case of the exact DW solution given by Eqs. (36) and (37), this expression takes an explicit form,

$$
U_{\text {int }}(L)=-4(\mu-\kappa)^{3 / 2} \exp (-2 \sqrt{\mu-\kappa} L) .
$$


A simple but essential property of expressions 45 and 46 is that they obviously predict attraction between the two DWs.

With the external potential $W(x)$ included into Eqs. (39), Hamiltonian density (4) is modified as

$$
\begin{gathered}
\mathcal{H}=\frac{1}{2}\left[\left(\phi_{1}^{\prime}\right)^{2}+\left(\phi_{2}^{\prime}\right)^{2}\right]+\frac{\sigma}{2}\left(\phi_{1}^{4}+\phi_{2}^{4}\right) \\
+g \phi_{1}^{2} \phi_{2}^{2}-2 \kappa \phi_{1} \phi_{2}+2 W(x)\left(\phi_{1}^{2}+\phi_{2}^{2}-A_{1}^{2}-A_{2}^{2}\right),
\end{gathered}
$$

where constants $A_{1,2}^{2}$ are subtracted from $\phi_{1,2}^{2}(x)$ for convenience (to cancel a formally diverging constant term in the Hamiltonian), $A_{1,2}$ being the same as in Eqs. (7). This means that the energy of the interaction of the DW with the external potential is

$$
U_{\text {pot }}=2 \int_{-\infty}^{+\infty} W(x)\left[\phi_{1}^{2}(x)+\phi_{2}^{2}(x)-A_{1}^{2}-A_{2}^{2}\right] d x \text {. }
$$

Assuming that $W(x)$ represents a broad potential barrier or well with a width much larger than the thickness of the DW, and treating the external potential as a perturbation (i.e., neglecting the distortion of the solution under the action of the potential), the substitution of the exact DW solution given by Eqs. (36) and (37) into Eq. (48) readily yields:

$$
U_{\text {pot }}(\xi) \approx-8 U_{1}^{2} \lambda^{-1} W(\xi) \equiv-2 \sqrt{\mu-\kappa} \sigma^{-1} W(\xi)
$$

where $\xi$ is the coordinate of the center of the DW. A straightforward consequence of Eq. 49 is that, as conjectured above, the DW tends to be trapped at local maxima of the external potential $W(x)$, as, due to sign minus in Eq. (49), they correspond to minima of the effective potential 449 .

If two DWs are trapped at two particular maxima of $W(x), \xi_{1}$ and $\xi_{2}$, separated by large distance $L$, the equilibrium condition for each DW is the vanishing of the total force produced by the interaction of the DW with its counterpart and with the external potential:

$$
\begin{aligned}
& \frac{\partial}{\partial \xi_{1}}\left[\frac{2 \sqrt{\mu-\kappa}}{\sigma} W\left(\xi_{1}\right)+4(\mu-\kappa)^{3 / 2} \exp \left(-2 \sqrt{\mu-\kappa}\left(\xi_{2}-\xi_{1}\right)\right)\right] \\
= & \frac{\partial}{\partial \xi_{2}}\left[\frac{2 \sqrt{\mu-\kappa}}{\sigma} W\left(\xi_{2}\right)+4(\mu-\kappa)^{3 / 2} \exp \left(-2 \sqrt{\mu-\kappa}\left(\xi_{2}-\xi_{1}\right)\right)\right]=0 .
\end{aligned}
$$

For example, if the potential is periodic, $W(x)=\epsilon \cos (2 \pi x / \Lambda)$, with large period $\Lambda$, one may consider the pair of DWs trapped at two adjacent potential maxima. Substituting this potential into Eqs. (50), it is easy to check that its minimum strength, necessary for holding the DW pair (i.e., preventing it from merger due to the mutual attraction) is $\epsilon_{\min }=(2 / \pi) \sigma \Lambda(\mu-\kappa)^{3 / 2} \exp (-\sqrt{\mu-\kappa} \Lambda)$.

\section{Numerical results for the model with an external potential}

The above analysis predicted that two DWs created in the free space attract each other. Direct simulations confirm the prediction, see a typical example in Fig. 4. Eventually, the two DWs annihilate into the stable uniform asymmetric state. Naturally, for larger initial values of the separation between the DWs, the attraction force is weaker, and a considerably longer time is required for the DW pair to manifest the interaction.

In the presence of the external potential in Eqs. (39), the numerical investigation was carried out for two different shapes of $W(x)$. First, the single potential barrier was considered, taken in the same form 40 which was used to obtain the exact solution. The corresponding stationary DW solutions are similar to their counterparts found in the free space. Specifically, out of the four types of the DWs categorized by the values of $\left(\phi_{1}(x), \phi_{2}(x)\right)$ at $x= \pm \infty$ as per Eq. (27), only the first one is stable, see a typical example in Fig. 5(a). In addition to the DWs, the potential barrier supports states in the form of "bubbles" (defined as per Ref. [29]), i.e., patterns with identical values of $\left(\phi_{1}(x), \phi_{2}(x)\right.$ ) at $x= \pm \infty$, and localized perturbations of $\phi_{1,2}(x)$ around the barrier, see Figs. 5(b), (c). Naturally, the bubbles supported by the asymmetric and symmetric CWs are stable and unstable, respectively.

The expectation that the DW is stably trapped by the potential peak is confirmed by direct simulations displayed in Fig. 6(a), for the DW of the first type, in terms of Eq. (27), i.e. $\left\{\left(A_{1} A_{2}\right),\left(A_{2} A_{1}\right)\right\}$ [it is the same DW which is displayed in Fig. 5(a)]. The DW, if shifted from the potential maximum by $\Delta x=5$, performs decaying oscillations around the peak. The decay of the oscillations is explained by emission of radiation waves into the background by the oscillating DW. 

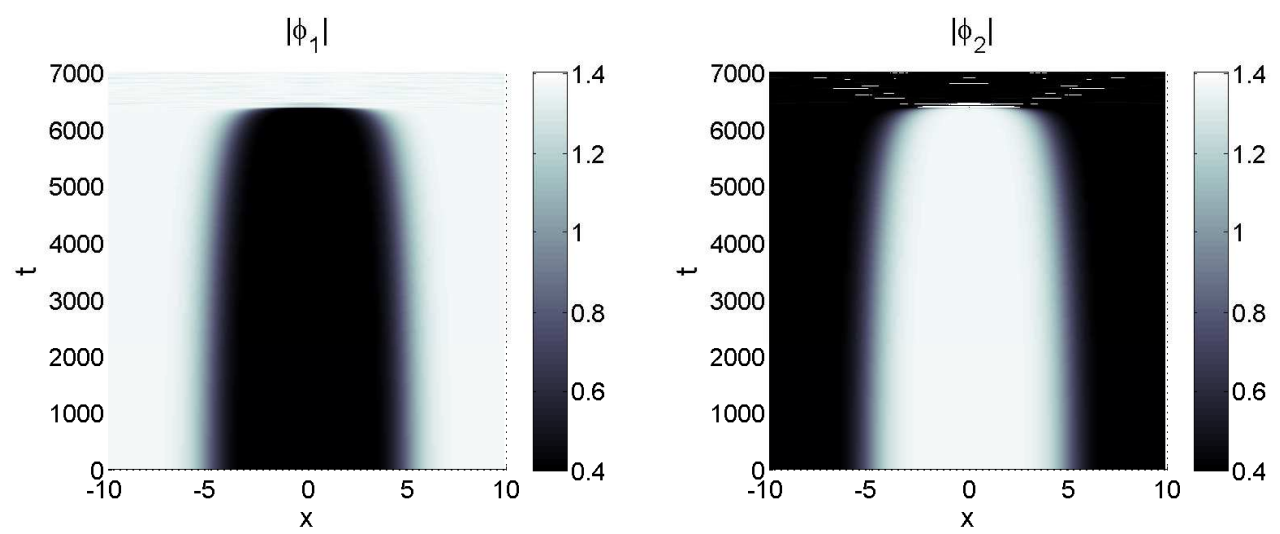

FIG. 4. The simulated evolution of a pair of identical domain walls, initially separated by distance $2 \Delta x=10$, is displayed by means of density contour plots, for $g=3, \sigma=1, \kappa=1$, and $\mu=2$.
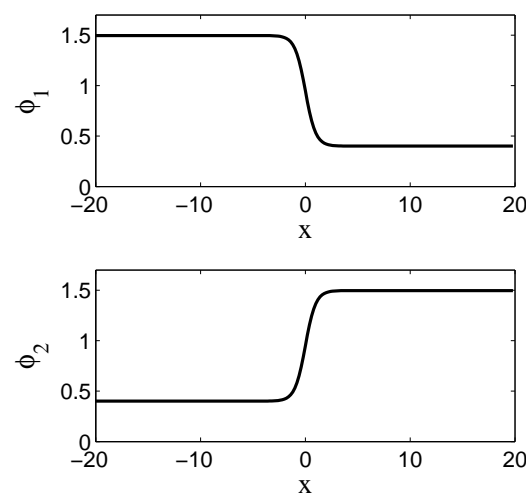

(a)
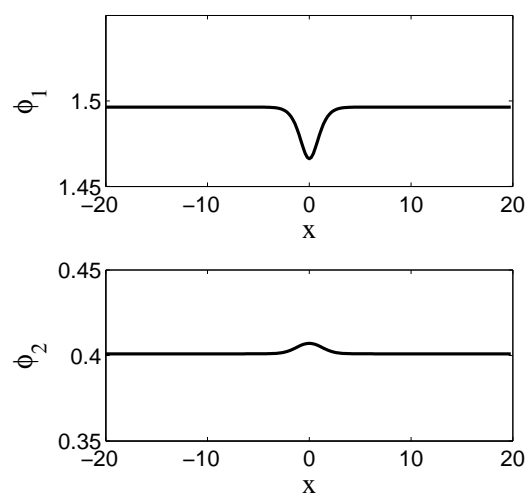

(b)
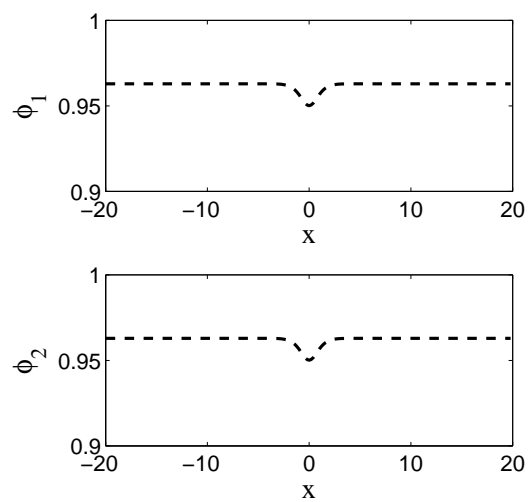

(c)

FIG. 5. Typical profiles of the domain-wall (a) and "bubble" (b,c) stationary solutions found in the presence of the potential peak 40, for $g=8 / 3, \sigma=1, \kappa=1, \lambda=1, W_{0}=0.1$, and $\mu=2.4$ [these values of the parameters admit the existence of the exact DW solution (41)- 42, which is actually displayed in (a)]. The stable and unstable solutions are depicted by continuous and dashed curves, respectively.

The attraction of DWs to the potential peak is further illustrated by Fig. 5(b), which displays a pair of identical DWs symmetrically placed, at $t=0$, at distances $\Delta x= \pm 5$ from the peak. Due to the attraction of both DWs to the peak, in this case their annihilation happens much sooner than for the same pair in the free space, cf. Fig. 4 for the same value of separation $2 \Delta x$ between the DWs.

The numerical analysis was also carried out for the double-peak potential, taken in the form of

$$
W(x)=W_{0}(2 x / L)^{2} \exp \left(1-(2 x / L)^{2}\right),
$$

where $W_{0}$ is the height of the two peaks and $L$ is the distance between them. The numerical solution of Eqs. (39) with potential (51) has revealed multiple structures, which may be considered as transient layers between CW states filling the three regions separated by the two potential barriers. These structures can be categorized into two groups, built of symmetric or asymmetric CWs. The structures of the former type are arranged as the following sets of the CW states in the three regions [cf. Eq. [27]]:

$$
\begin{gathered}
\left\{\left(A_{0}, A_{0}\right),\left(A_{0}, A_{0}\right),\left(A_{0}, A_{0}\right)\right\} ;\left\{\left(A_{0}, A_{0}\right),\left(A_{0}, A_{0}\right),\left(-A_{0},-A_{0}\right)\right\} ; \\
\left\{\left(A_{0}, A_{0}\right),\left(-A_{0},-A_{0}\right),\left(A_{0}, A_{0}\right)\right\} .
\end{gathered}
$$

These three varieties may be interpreted, respectively, as containing none, one, or two dark solitons trapped in each component. 

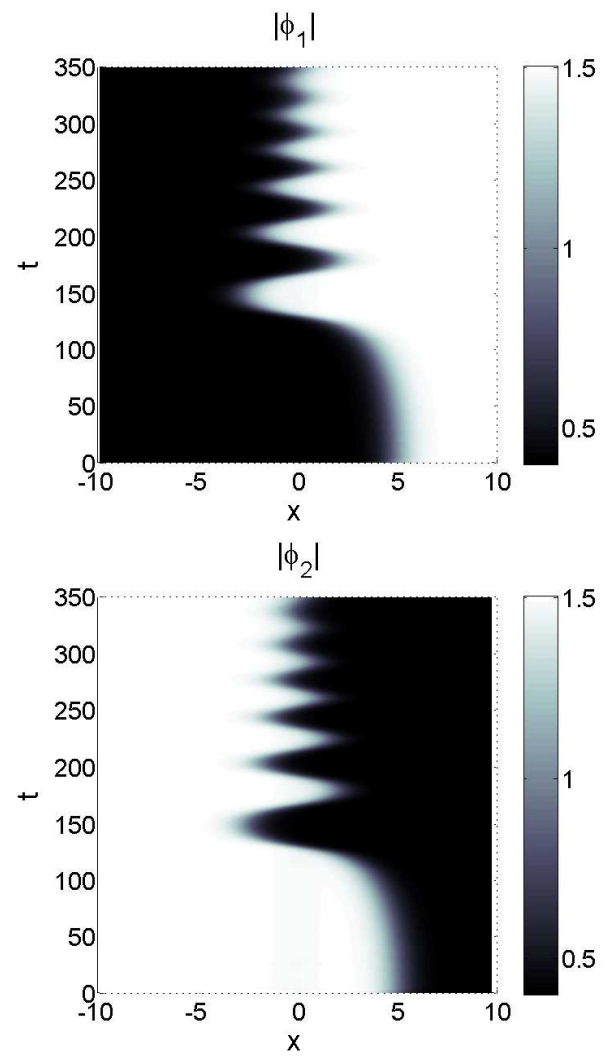

(a)
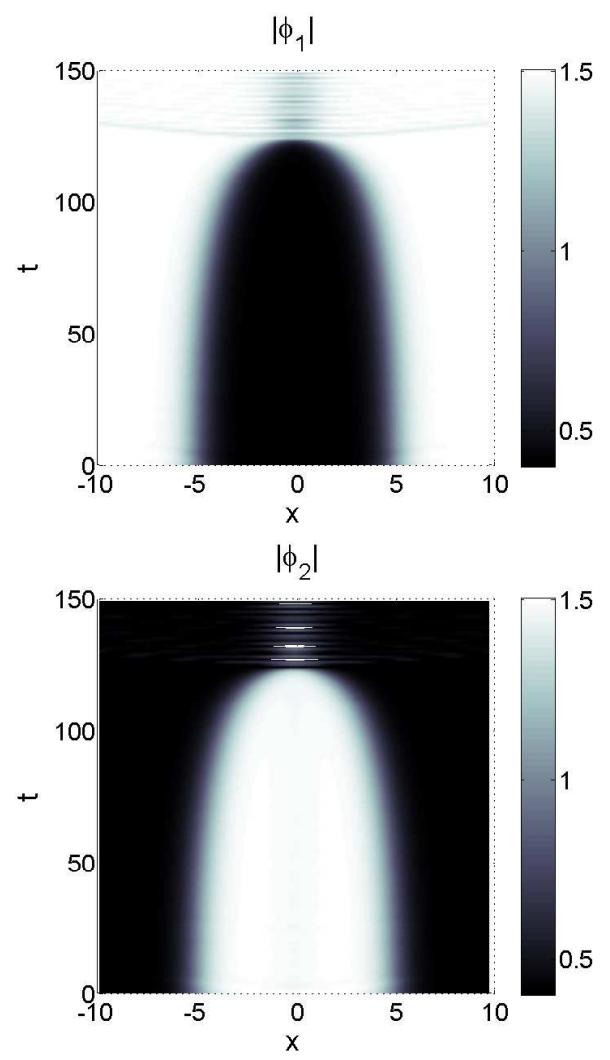

(b)

FIG. 6. (a) The evolution of the domain wall shifted by $\Delta x=5$ from the maximum of potential barrier 40 , for the same parameters as in Fig.5(a). (b) Similar to panel (a), but for the pair of DWs, placed symmetrically on both sides of the potential maximum, at $x= \pm 5$.

Using various combinations of the asymmetric CW states, we have built the following patterns supported by the double-peak potential, cf. Eqs. (27) and (52):

$$
\begin{gathered}
\left\{\left(A_{1}, A_{2}\right),\left(A_{1}, A_{2}\right),\left(A_{1}, A_{2}\right)\right\} ;\left\{\left(A_{1}, A_{2}\right),\left(A_{1}, A_{2}\right),\left(A_{2}, A_{1}\right)\right\} ; \\
\left\{\left(A_{1}, A_{2}\right),\left(A_{2}, A_{1}\right),\left(A_{1}, A_{2}\right)\right\} ; \\
\left\{\left(A_{1}, A_{2}\right),\left(A_{1}, A_{2}\right),\left(-A_{1},-A_{2}\right)\right\} ;\left\{\left(A_{1}, A_{2}\right),\left(-A_{1},-A_{2}\right),\left(A_{1}, A_{2}\right)\right\} ; \\
\left\{\left(A_{1}, A_{2}\right),\left(A_{1}, A_{2}\right),\left(-A_{2},-A_{1}\right)\right\} ;\left\{\left(-A_{1},-A_{2}\right),\left(A_{1}, A_{2}\right),\left(A_{2}, A_{1}\right)\right\} ; \\
\left\{\left(A_{1}, A_{2}\right),\left(-A_{1},-A_{2}\right),\left(A_{2}, A_{1}\right)\right\} ;\left\{\left(A_{1}, A_{2}\right),\left(A_{2}, A_{1}\right),\left(-A_{1},-A_{2}\right)\right\} ; \\
\left\{\left(A_{1}, A_{2}\right),\left(-A_{2},-A_{1}\right),\left(A_{1}, A_{2}\right)\right\} .
\end{gathered}
$$

The three patterns (53) feature, respectively, none, one, or two trapped DWs, while seven patterns (54) include dark solitons or zero-crossing (sign-changing) DWs. As before, those patterns which do not cross zero in any component are stable [in the case of the first pattern in (52), this is, naturally, true prior to the SBB], while all the solutions featuring at least one zero crossing are unstable. Examples of the patterns of these types are presented in Fig. 7, for $W_{0}=0.1$ and $L=10$.

The attractive interaction of the DWs with the potential peaks is additionally illustrated by simulations displayed in Fig. 8. In particular, if a single DW is initially placed at the midpoint between the peaks, the evolution of this obviously unstable configuration leads to damped oscillations of the DW around either peak to which it is pulled, see Fig. 8(a). On the other hand, symmetrically placing pairs of identical DWs either between (Fig. 8(b) or outside (Fig. 8(c) of the potential peaks, we observe coherent oscillations with the apparently unbroken symmetry. In the latter case, the symmetric oscillations feature very slow damping, which is explained by the small value of $W_{0}=0.1 \mathrm{in}$ this case. Similar simulations for larger $W_{0}$ demonstrate oscillations which converge much faster to a stable symmetric configuration, with each DW trapped by one potential peak. 

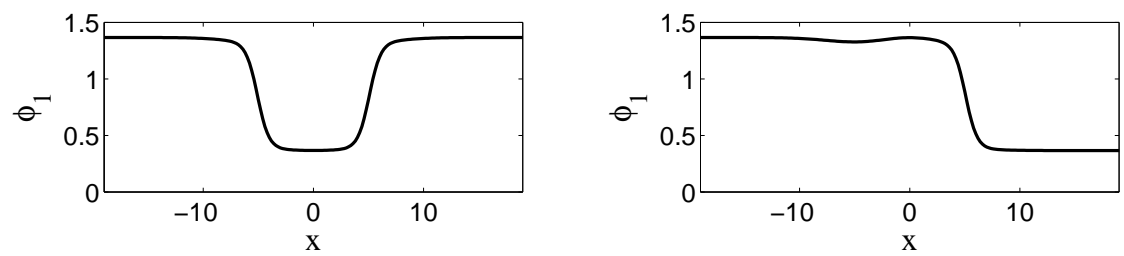

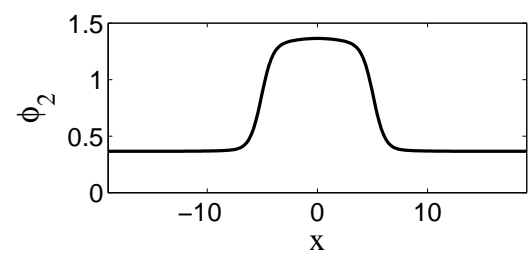

(a)
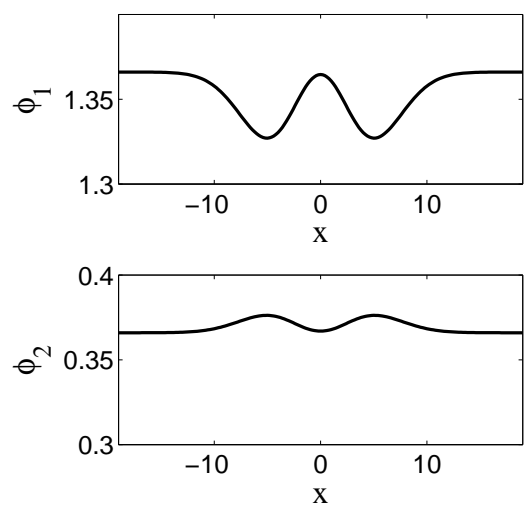

(c)

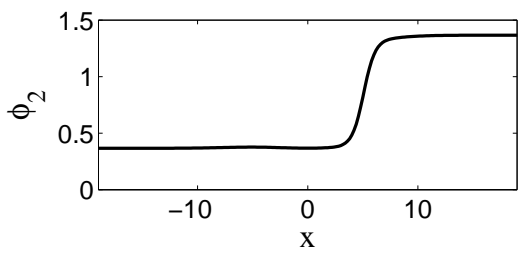

(b)
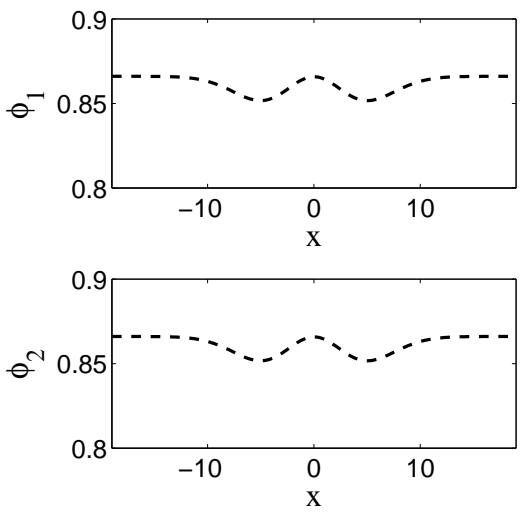

(d)

FIG. 7. Typical examples of patterns supported by the double-barrier potential (51), with $W_{0}=0.1$ and $L=10$, constants $g, \sigma$, $\kappa$ and $\mu$ being the same as in Fig. 4 . Panels (a), (b) and (c) demonstrate the stable patterns of the following types, in terms of Eq. (53): $\left\{\left(A_{1}, A_{2}\right),\left(A_{2}, A_{1}\right),\left(A_{1}, A_{2}\right)\right\},\left\{\left(A_{1}, A_{2}\right),\left(A_{1}, A_{2}\right),\left(A_{2}, A_{1}\right)\right\}$, and $\left\{\left(A_{1}, A_{2}\right),\left(A_{1}, A_{2}\right),\left(A_{1}, A_{2}\right)\right\}$, respectively. The unstable pattern of type $\left\{\left(A_{0}, A_{0}\right),\left(A_{0}, A_{0}\right),\left(A_{0}, A_{0}\right)\right\}$, from set [52), is presented in panel (d).

\section{VORTICES AND TWO-DIMENSIONAL CIRCULAR DOMAIN WALLS}

The $2 \mathrm{D}$ version of model (1) is

$$
\begin{aligned}
& i\left(\psi_{1}\right)_{t}=-(1 / 2)\left[\left(\psi_{1}\right)_{x x}+\left(\psi_{1}\right)_{y y}\right]+\sigma\left|\psi_{1}\right|^{2} \psi_{1}+g\left|\psi_{2}\right|^{2} \psi_{1}-\kappa \psi_{2}, \\
& i\left(\psi_{2}\right)_{t}=-(1 / 2)\left[\left(\psi_{2}\right)_{x x}+\left(\psi_{2}\right)_{y y}\right]+\sigma\left|\psi_{2}\right|^{2} \psi_{2}+g\left|\psi_{1}\right|^{2} \psi_{2}-\kappa \psi_{1} .
\end{aligned}
$$

In terms of BEC, Eqs. (55) admit the straightforward interpretation as the GP equations for the two-component condensate in a $2 \mathrm{D}$ pancake-shaped configuration. In terms of optics, these equations, with $t$ replaced by propagation coordinate $z$, govern the transmission of a stationary beam through the bulk nonlinear medium, with functions $\psi_{1,2}$ representing two circular polarizations. In the latter case, the linear mixing between the polarizations can be induced by the linear birefringence, which, in turn, may be imposed by mechanical stress applied perpendicular to the propagation axis [8], or, alternatively, by dc magnetic field applied in the same direction [30] (the birefringence imposed by the transverse magnetic field leads to the classical Cotton-Mouton/Voigt effects 31]).

General axisymmetric solutions to Eqs. (55) are looked for in the form of the optical vortices (alias 2D dark solitons) [8, 32, which also correspond to the vortex modes in BEC [9]:

$$
\psi_{1,2}(x, y, t)=\phi_{1,2}(r, t) \exp (i s \theta) \exp (-i \mu t)
$$

where $r$ and $\theta$ are the polar coordinates in the $(x, y)$ plane, and integer $s$ is the topological charge (vorticity, alias "spin"). 


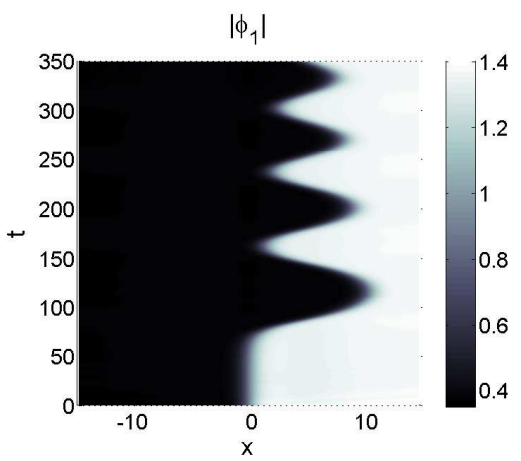

$\left|\phi_{2}\right|$

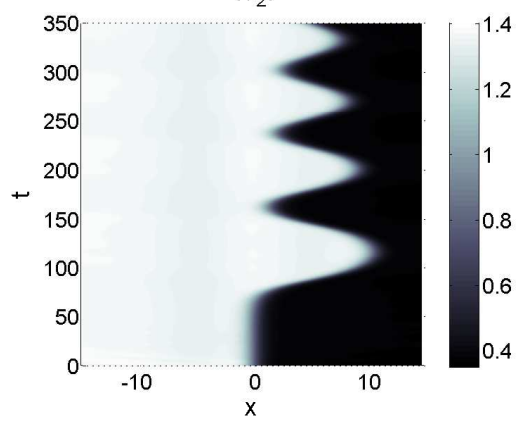

(a)

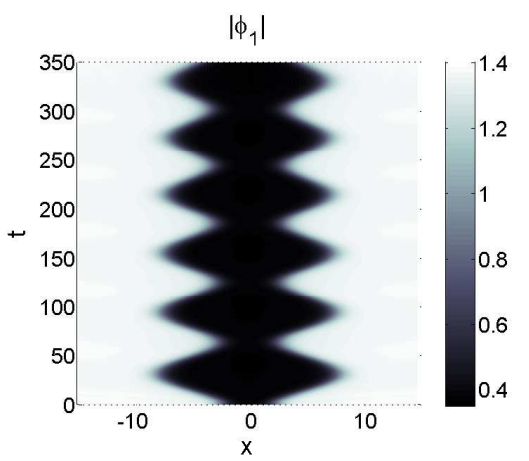

$\left|\phi_{2}\right|$

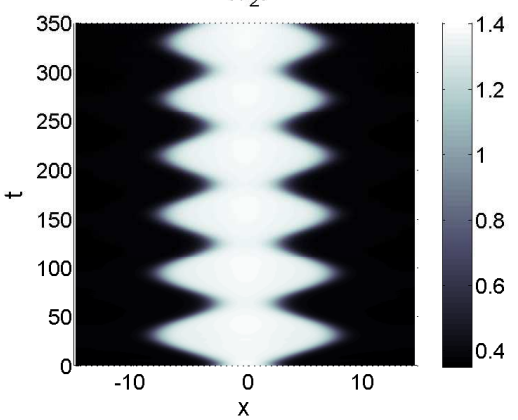

(b)

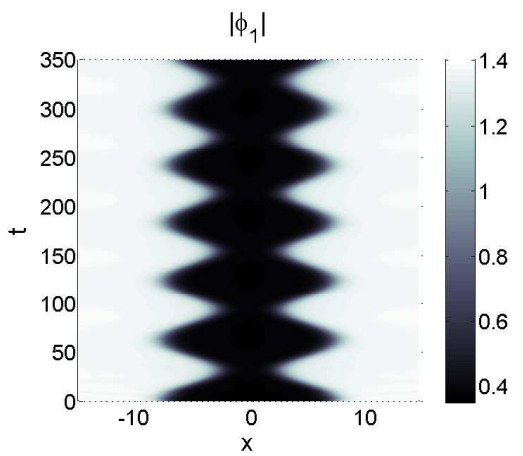

$\left|\phi_{2}\right|$

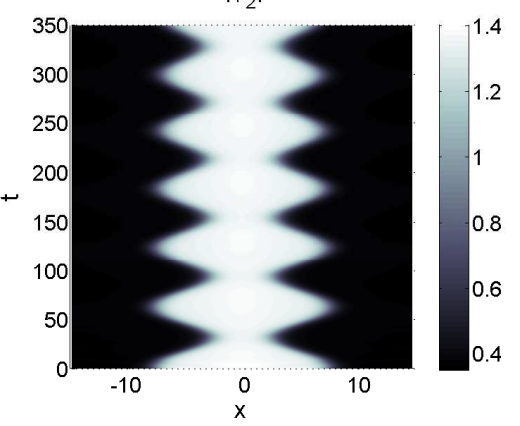

(c)

FIG. 8. The evolution of a single DW and pairs of DWs in the presence of the double-peak external potential, (51), with $W_{0}=0.1$ and $L=10$. The other parameters are as in Figs. 4 and 7 In panel (a), the single DW is initially positioned exactly between the two peaks. The evolution of pairs of DWs, symmetrically placed at $x= \pm 2.5$ and $x= \pm 7.5$, is displayed in panels (b) and (c), respectively.

The substitution of expressions (56) into Eqs. (55) leads to the equations for radial wave functions $\phi_{1,2}(r, t)$ :

$$
\begin{gathered}
i\left(\phi_{1}\right)_{t}+\mu \phi_{1}+(1 / 2)\left[\left(\phi_{1}\right)_{r r}+r^{-1}\left(\phi_{1}\right)_{r}-s^{2} r^{-2} \phi_{1}\right] \\
-\sigma\left(\phi_{1}\right)^{3}-g\left(\phi_{2}\right)^{2} \phi_{1}+\kappa \phi_{2}=0, \\
i\left(\phi_{2}\right)_{t}+\mu \phi_{2}+(1 / 2)\left[\left(\phi_{2}\right)_{r r}+r^{-1}\left(\phi_{2}\right)_{r}-s^{2} r^{-2} \phi_{2}\right] \\
-\sigma\left(\phi_{2}\right)^{3}-g\left(\phi_{1}\right)^{2} \phi_{2}+\kappa \phi_{1}=0 .
\end{gathered}
$$

Obviously, stationary CW solutions in the 2D model are identical to those obtained for the 1D model, see Eqs. (5) and (7). In particular, the bifurcation described by Eqs. (10) and (11) is relevant in the 2D case too.

The study of the existence, stability and dynamics of 2D axisymmetric patterns, generated by Eqs. (57), is presented below in two steps. First, stationary vortices, supported by the asymmetric CW background, are obtained for spins $s=0,1,2$ and 3 , and their stability is determined. Then, the evolution of the pulsons, i.e., circular DWs oscillating in the radial direction, is investigated by means of direct simulations. In this connection, it is relevant to mention that patterns in the form of circular DWs are well known in various magnetic media [33]

\section{A. Stationary vortices}

To find stationary solutions of Eqs. (57), we proceed to the time-independent version of these equations and apply the Newton-Raphson method to the respective nonlinear boundary-value problem. The boundary conditions demand that $\phi_{1,2}(r) \rightarrow r^{s}$ at $r \rightarrow 0$, while, at $r \rightarrow \infty, \phi_{1,2}(r)$ asymptotically approach the symmetric or asymmetric CW solutions, (5) or (7). Examples of the radial profiles of such modes are displayed in Fig. 9. For the $s=0$, no stationary solutions exist, apart from the obvious flat states (which are not shown in Fig. 9). For each non-zero value of the spin that was examined, $s=1,2$ and 3 , two families of nontrivial stationary solutions were obtained, supported [past bifurcation point [10] ] by the symmetric and asymmetric CW states at $r \rightarrow \infty$, hence they demonstrate precisely the same existence and bifurcation features as the corresponding flat states, (5) and (7). 


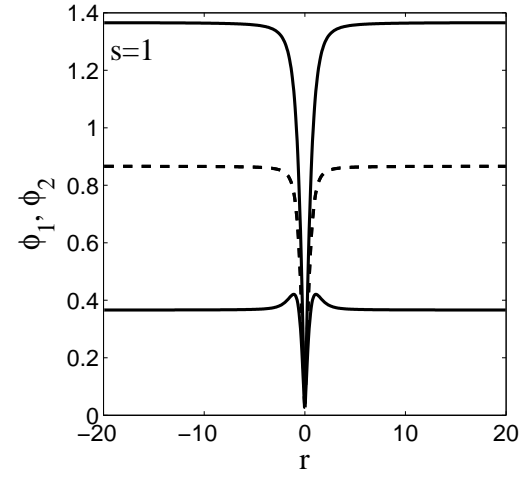

(a)

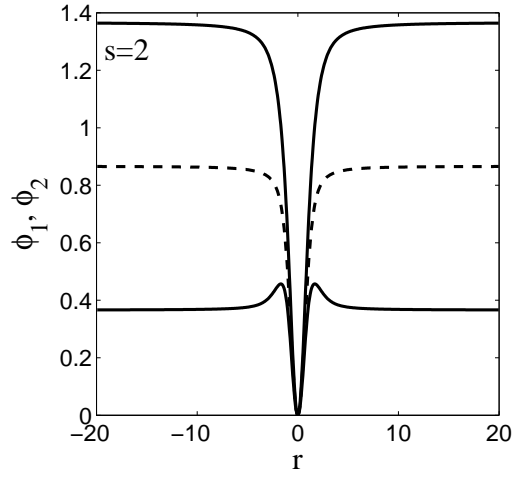

(b)

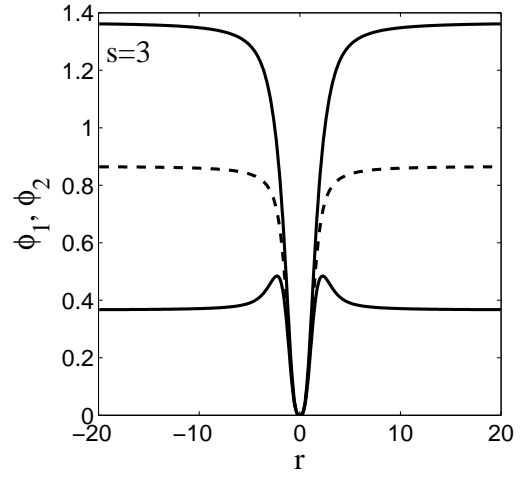

(c)

FIG. 9. (a,b,c): Radial profiles of the symmetric and asymmetric stationary vortices, with spin $s=1,2$, and 3 , respectively (no non-flat stationary 2D solutions were found for $s=0$ ). The parameters are $g=3, \sigma=1, \kappa=1$, and $\mu=2$. Solid and dashed curves depict, respectively, two components of the stable asymmetric solutions, and unstable symmetric ones.

To explore the stability of the 2D stationary solutions, we seek for perturbed solutions as [cf. Eqs. [28]]

$$
\begin{aligned}
& \widetilde{\phi_{1}}(r, \theta, t)=U(r)+\left[U_{+}(r) \exp (i n \theta)+U_{-}(r) \exp (-i n \theta)\right] \exp \left(\gamma_{n} t\right), \\
& \widetilde{\phi_{2}}(r, \theta, t)=V(r)+\left[V_{+}(r) \exp (i n \theta)+V_{-}(r) \exp (-i n \theta)\right] \exp \left(\gamma_{n} t\right),
\end{aligned}
$$

where integer $n>0$ is an arbitrary azimuthal index of the perturbation, and $\gamma_{n}$ is the corresponding instability growth rate. After substituting expressions (58) into Eqs. (57) and linearizing, the following system of equations is obtained:

$$
\begin{gathered}
\mu U_{+}+i \gamma_{n} U_{+}+(1 / 2) U_{+}^{\prime \prime}+(2 r)^{-1} U_{+}^{\prime}-(s+n)^{2}\left(2 r^{2}\right)^{-1} U_{+} \\
-g U V\left(V_{-}^{*}+V_{+}\right)-\sigma U^{2}\left(U_{-}^{*}+2 U_{+}\right)-g V^{2} U_{+}+\kappa V_{+}=0 ; \\
\mu U_{-}+i \gamma_{n} U_{-}+(1 / 2) U_{-}^{\prime \prime}+(2 r)^{-1} U_{-}^{\prime}-(s-n)^{2}\left(2 r^{2}\right)^{-1} U_{-} \\
-g U V\left(V_{+}^{*}+V_{-}\right)-\sigma U^{2}\left(U_{+}^{*}+2 U_{-}\right)-g V^{2} U_{-}+\kappa V_{-}=0 ; \\
\mu V_{+}+i \gamma_{n} V_{+}+(1 / 2) V_{+}^{\prime \prime}+(2 r)^{-1} V_{+}^{\prime}-(s+n)^{2}\left(2 r^{2}\right)^{-1} V_{+} \\
-g U V\left(U_{-}^{*}+U_{+}\right)-\sigma V^{2}\left(V_{-}^{*}+2 V_{+}\right)-g U^{2} U_{+}+\kappa U_{+}=0 ; \\
\mu V_{-}+i \gamma_{n} V_{-}+(1 / 2) V_{-}^{\prime \prime}+(2 r)^{-1} V_{-}^{\prime}-(s-n)^{2}\left(2 r^{2}\right)^{-1} V_{-} \\
-g U V\left(U_{+}^{*}+U_{-}\right)-\sigma V^{2}\left(V_{+}^{*}+2 V_{-}\right)-g U^{2} V_{-}+\kappa U_{-}=0
\end{gathered}
$$

where the prime stands for $d / d r$. We treat Eqs. (59) as an algebraic eigenvalue problem for $\gamma_{n}$ and solve it directly, using a finite-difference method. The largest instability-growth rate is identified as the real part of the most unstable eigenvalue, $\max \left\{\operatorname{Re}\left(\gamma_{n}\right)\right\}$. Following this approach, we have confirmed that the only source of the destabilization is the transition between the symmetric and asymmetric modes, which, as said above, is actually driven by the SBB in the flat background at $r \rightarrow \infty$. In particular, no azimuthal instability, that would break the axial symmetry, was found for $s=1,2,3$, for all integer values of $n$ considered. The absence of the azimuthal instability in the case of the self-defocusing nonlinearity is actually a natural feature 34. In addition, this finding implies that the flat (quasi-one-dimensional) DW is stable against corrugations in the 2D setting.

\section{B. Direct simulations - shrinking domain walls and pulsons}

Another aspect of the 2D model based on equations (55) was examined by investigating the development of circular DWs into pulsons, i.e., annular grain boundaries periodically shrinking and expanding in the radial directions. It is known that, while exact solutions for such pulsons do not exist, in some models, such as the 2D single-component sine-Gordon equation, the pulsons may be remarkably stable, featuring hundreds 35, 37] and thousands 36 of radial pulsations with very little loss. 

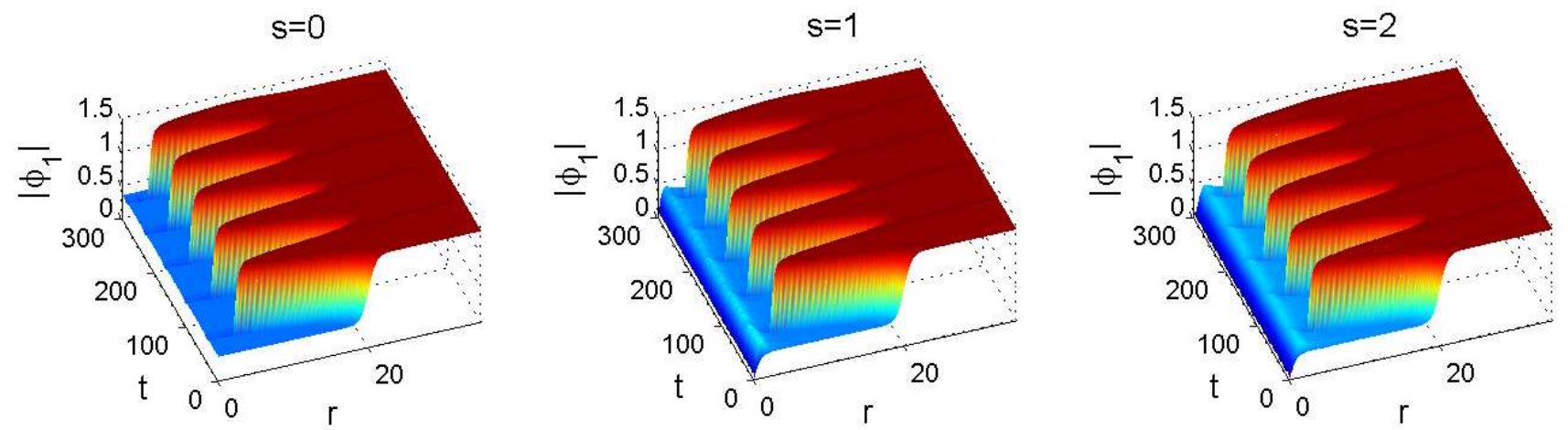

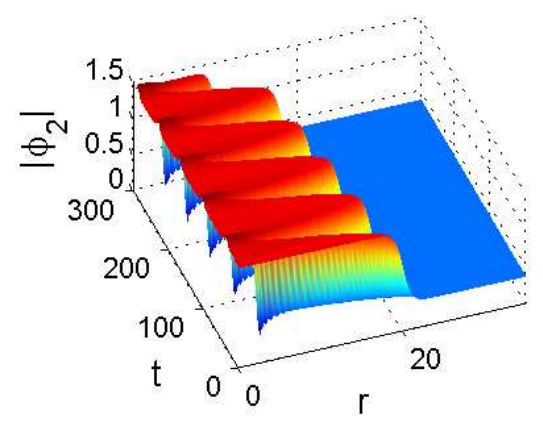

(a)

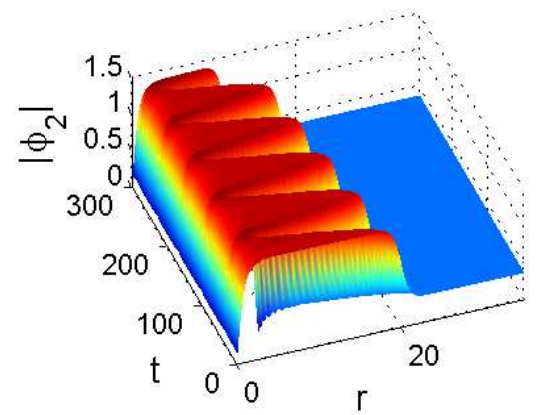

(b)

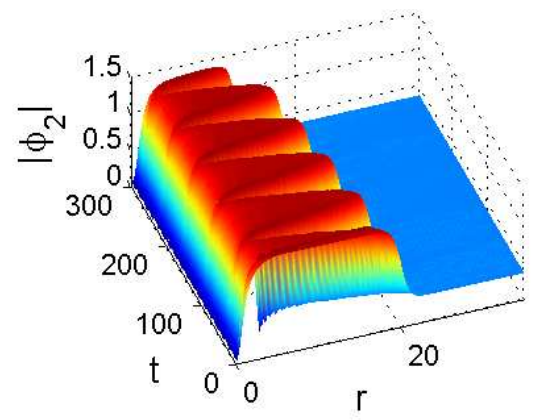

(c)

FIG. 10. The initial evolution of the pulsons (circular domain walls) for $s=0$ (a), $s=1$ (b), and $s=2$ (c). The initial radius of the circular DW is $R_{0}=20$, the other parameters being $g=3, \sigma=1, \kappa=1$ and $\mu=2$.

We have performed simulations of pulsating radial DWs for three values of the spin, $s=0,1$ and 2 . The simulations were implemented by means of the linearized Crank-Nicolson scheme. As initial conditions, the following DW configuration, based on the asymmetric uniform states (7), was used,

$$
\begin{aligned}
& \phi_{1}(r, t=0)=(1 / 2) \tanh ^{s}(r)\left\{A_{1}\left[1+\tanh \left(r-R_{0}\right)\right]+A_{2}\left[1-\tanh \left(r-R_{0}\right)\right]\right\}, \\
& \phi_{2}(r, t=0)=(1 / 2) \tanh ^{s}(r)\left\{A_{1}\left[1-\tanh \left(r-R_{0}\right)\right]+A_{2}\left[1+\tanh \left(r-R_{0}\right)\right]\right\},
\end{aligned}
$$

where $R_{0}$ is the initial radius of the DW. Note that this initial ansatz complies with the necessary boundary condition at $r \rightarrow 0, \phi_{1,2}(r) \sim r^{s}$. The boundary conditions at the right edge of the integration interval, $0<r<\rho$, were adopted as $\left.\left(\partial \phi_{1,2} / \partial r+\partial \phi_{1,2} / \partial t\right)\right|_{r=\rho}=0$. These conditions prevent the reflection of the emitted radiation from the boundary.

For each value of the spin considered here, $s=0,1,2$, the simulations were run for several values of the initial radius, $R_{0}$. Typical examples of the initial stage of the observed evolution are demonstrated in Fig. 10 , for $R_{0}=20$ and for the parameters $g=3, \sigma=1, \kappa=1$ and $\mu=2$. Similar results were obtained for other values of $R_{0}$.

It was observed that, while the pulsons shrink and expand in a quasi-periodic manner, their smallest and largest radii, $R_{\min }$ and $R_{\max }$, do not remain constant, slowly decreasing from a cycle to a cycle, as shown in Fig. 11. In particular, the decay of $R_{\max }$ is roughly exponential in time, with some irregularities observed at $t \simeq 400$ and at the final stage of the evolution. The decay is plausibly caused by the emission of radiation waves by the pulsating DW.

Also decreasing is the oscillation period, $\tau_{l}$, taken as the time interval between two consecutive points at which the pulson expands to the largest radius, $\tau_{l}=t\left(r=R_{\max , l}\right)-t\left(r=R_{\max , l-1}\right)$, where $l$ is the number of the cycle. The relation between the slowly decreasing period and the effective maximum radius, $R_{\max , \text { eff }} \equiv\left(R_{\max , l}+R_{\max , l-1}\right) / 2$, is presented in Fig. 12 The plots demonstrate a nearly linear dependence for all $s$. The same linear dependence was obtained for different values of the initial radius, including $R_{0}=10,15,30$, and 40 .

The linear relation between $\tau_{l}$ and $R_{\max }$ can be easily explained. Indeed, considering a large-radius circular DW, with radius $R$ much larger than the thickness of the DW in the radial direction, one can define the effective mass for the radial pulsations, $M=2 \pi R m$, and the effective surface-tension energy, $E_{\mathrm{ST}}=2 \pi R \alpha$, where $m$ and $\alpha$ are the 


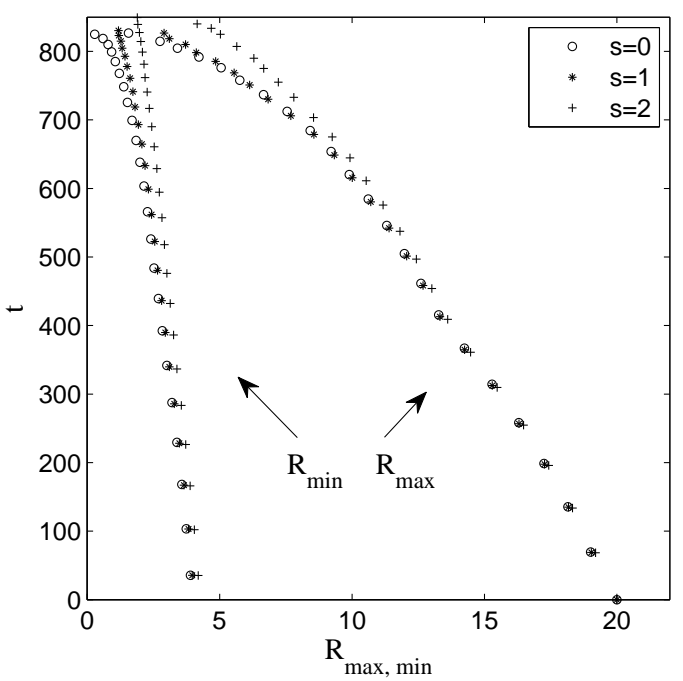

FIG. 11. The temporal decay of the largest and smallest radii of the pulson, in the cases shown in Fig. 10 , for $s=0,1$ and 2 .

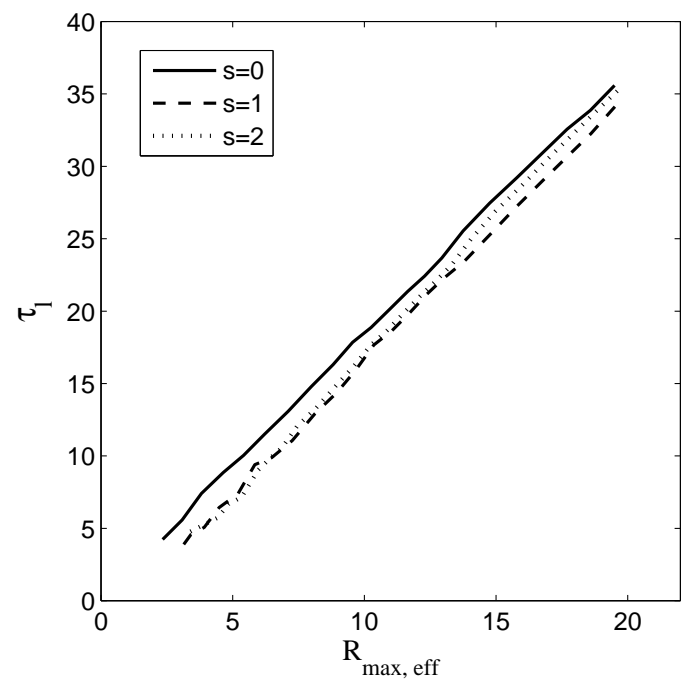

FIG. 12. The relation between the gradually decreasing oscillation period of the pulson, $\tau_{l}$, and the effective maximum radius, $R_{\max , \text { eff }}$, for $s=0,1$ and 2. The parameters are the same as in Fig. 10.

effective mass and surface-energy densities of the quasi-one-dimensional DW. Thus, the Newton's equation of motion for the DW in the radial direction is

$$
\frac{d}{d t}\left(2 \pi m R \frac{d R}{d t}\right)=-\frac{d}{d R}(2 \pi \alpha R)
$$

from where the law of motion follows: $R^{2}(t)=R_{\max }^{2}-(\alpha / m) t^{2}$, assuming that the motion starts with the zero initial velocity and $R=R_{\max }$. According to this result, the shrinking DW ring will bounce back from the center at the moment of time $\tau_{l} / 2=\sqrt{\alpha / m} R_{\max }$, which is obviously equal to a half of the period. This result explains the linear proportionality of $\tau_{l}$ to $R_{\max }$.

Finally, Fig. 13 displays the final stage of the evolution, observed when the pulson's radius and oscillation period have been sufficiently reduced. In this case, the oscillatory behavior fades out and the pulsons transform into the stable asymmetric stationary states which are presented above in subsection VIA (in particular, they are flat for $s=0$, and feature the vortex shape for $s=1$ and 2). For the case considered above (with $R_{0}=20$ ), the transition to the eventual stationary state occurs at $t \approx 830$, for all the values of the spin. 

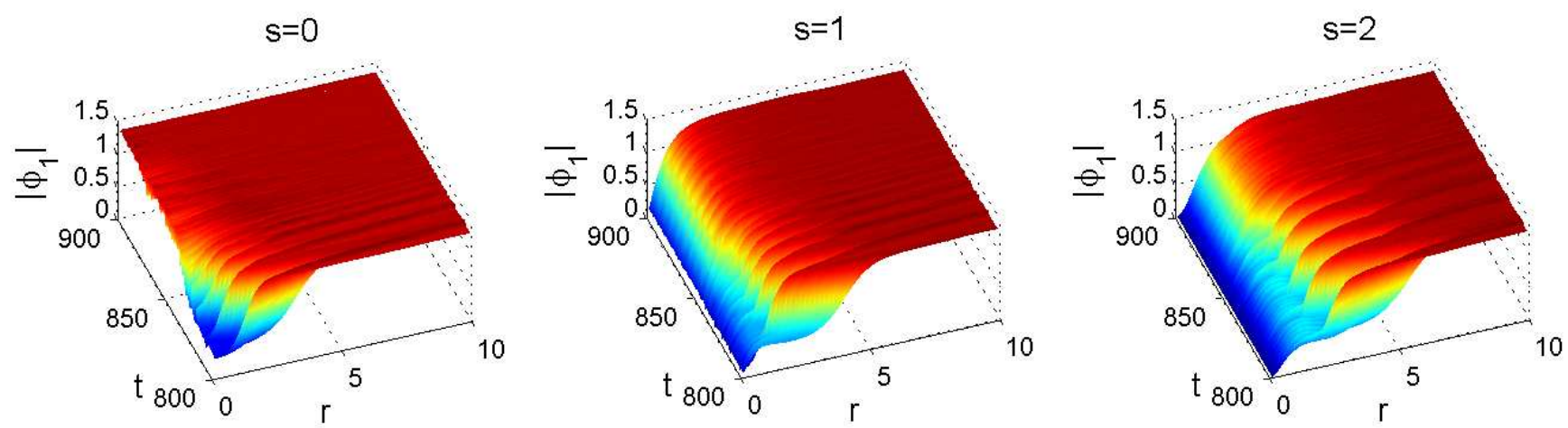

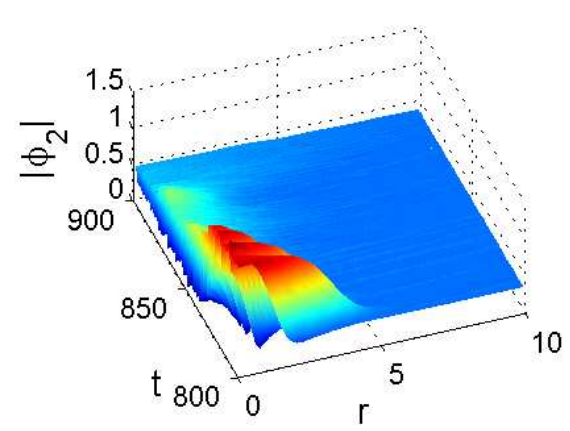

(a)

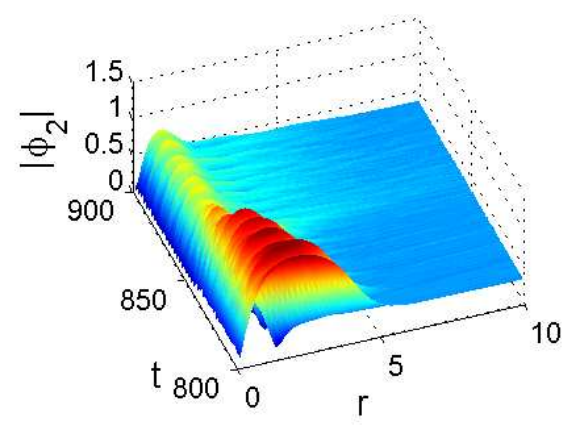

(b)

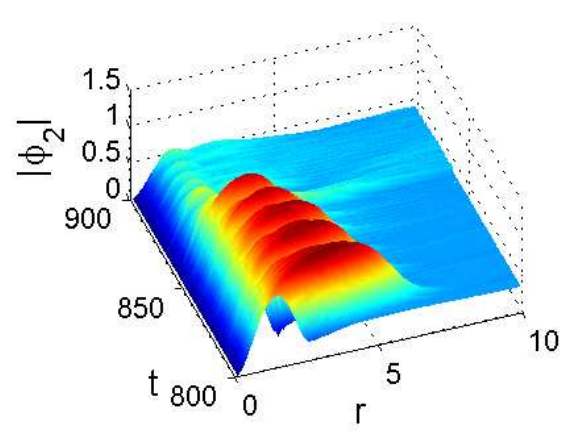

(c)

FIG. 13. (Color online) The final stage of the evolution of the pulsons with $s=0,1$ and 2, whose initial evolution was displayed in Fig. 10. The pulsons with $s=1$ and 2 transform into the asymmetric stationary (stable) modes shown in Fig. 9 .

\section{CONCLUSIONS}

The objective of this work was to investigate fundamental DW (domain-wall) modes in the system of nonlinearSchrödinger/Gross-Pitaevskii equations, coupled by the linear and XPM (cross-phase-modulation) terms. The system of coupled equations has natural realizations in two-component Bose-Einstein condensate, and in nonlinear optics, where the two wave functions represent amplitudes of co-propagating signals with orthogonal polarizations. First, conditions providing for the stability of the uniform CW (continuous wave) symmetric and asymmetric bimodal states, which support the DW patterns, were identified, and then the general families of DW solutions were constructed in the 1D setting. In particular, approximate analytical solutions were found near the symmetry-breaking bifurcation point of the CW states, and an exact solution was found for the XPM/SPM ratio $3: 1$. The DW states connecting asymptotically flat asymmetric states which are mirror images to each other (without the change of the overall sign) are stable, while all other types of the DWs, which feature zero crossings (including dark solitons), are unstable. Interactions between two DWs with opposite polarities were also considered. The potential of the attraction between them was found in the analytical form, and numerical simulations have demonstrated that the attraction leads to annihilation of the DW pair.

DWs trapped by the single or double potential peaks were investigated too. An exact solution for the DW trapped by a single peak was found. In the general case, it was predicted and corroborated by direct simulations that the bound state of the DW placed at a maximum of the external potential is stable. The interaction of two DWs in the presence of the single- and double-peak potentials was also studied.

The analysis was extended to axisymmetric patterns in the 2D geometry, including vortices carrying the topological charge $s=1,2,3$ and supported by the asymmetric flat background at infinity. Stable stationary states for the vorticity-carrying DW rings were found. The stability of the vortices against azimuthal perturbations was verified through the computation of the corresponding eigenvalues. Oscillations of annular DW-shaped pulsons were studied by means of systematic simulations (the linear relation between the period of the radial oscillations and largest radius of the annular DW was obtained in an analytical form). The evolution of the pulsons ends up with their relaxation 
into the stationary 2D modes (vortices, for $s \neq 0$, or simply the flat asymmetric state in the case of $s=0$ ).

This work may be extended by a more systematic investigation of the $2 \mathrm{D}$ system, without assuming the axial symmetry of the patterns. In particular, oscillations of eccentricity in elliptically deformed pulsons may be interesting to study, cf. Ref. 37]. In 1D, it may be also interesting to study in detail the behavior of DWs against the backdrop of periodic potentials (optical lattices), as well as in nonstationary systems with time-dependent parameters.

\section{ACKNOWLEDGMENT}

The work of J.Z. was supported, in a part, by a postdoctoral fellowship provided by the Tel Aviv University, and by grant No. 149/2006 from the German-Israel Foundation.

[1] V. G. Bar'yakhtar, M. V. Chetkin, B. A. Ivanov, and S. N. Gadetskii, Dynamics of Topological Magnetic Solitons (SpringerVerlag: Berlin, 1994).

[2] D. Damjanović, Rep. Progr. Phys. 61, 1267 (1998).

[3] P. G. de Gennes and J. Prost, The Physics of Liquid Crystals (Oxford University Press: New York, 1995).

[4] P. Manneville and Y. Pomeau, Phil. Mag. A 48, 607 (1983); G. Tesauro and M. C. Cross, ibid. 56, 703 (1987); T. Passot and A. C. Newell, Physica D 74, 301 (1994).

[5] E. Bodenschatz, W. Pesch, and G. Ahlers, Ann. Rev. Fluid Mech. 32, 709 (2000).

[6] A. A. Nepomnyashchy, M. G. Velarde, and P. Colinet, Interfacial Phenomena and Convection (Chapman and Hall/CRC: New York, 2001).

[7] B. A. Malomed, A. A. Nepomnyashchy, and M. I. Tribelsky, Phys. Rev. A 42, 7244 (1990).

[8] Y. S. Kivshar and G. P. Agrawal, Optical Solitons: From Fibers to Photonic Crystals (Academic Press: San Diego, 2003).

[9] L. Pitaevskii and S. Stringari, Bose-Einstein Condensation (Clarendon Press: Oxford, 2003).

[10] M. Haelterman and A. P. Sheppard, Phys. Lett. A 185, 265 (1994).

[11] B. A. Malomed, Phys. Rev. E 50, 1565 (1994).

[12] M. Trippenbach, K. Goral, K. Rzazewski, B. Malomed, and Y. B. Band, J. Phys. B: At. Mol. Opt. 33, 4017 (2000); P. Ohberg and L. Santos, Phys. Rev. Lett. 86, 2918 (2001); S. Coen and M. Haelterman, ibid. 87, 140401 (2001); J. J. Garcia-Ripoll, V. M. Pérez-García and F. Sols, Phys. Rev. A 66, 021602 (2002); P. G. Kevrekidis, H. E. Nistazakis, D. J. Frantzeskakis, B. A. Malomed, and R. Carretero-González, Eur. Phys. J. D 28, 181 (2004); B. A. Malomed, H. E. Nistazakis, D. J. Frantzeskakis, and P. G. Kevrekidis, Phys. Rev. A 70, 043616 (2004); J. Ruostekoski, ibid. 70, 041601(R) (2004); K. Kasamatsu and M. Tsubota, Phys. Rev. Lett. 93, 100402 (2004); K. Kasamatsu, M. Tsubota, and M. Ueda, ibid. 93, 250406 (2004); P. G. Kevrekidis, H. Susanto, R. Carretero-González, B. A. Malomed, and D. J. Frantzeskakis, Phys. Rev. E 72, 066604 (2005); Y.-C. Kuo, W.-W. Lin, and S.-F. Shieh, Physica D 211, 211 (2005).

[13] C. Kubstrup, H. Herrero, and C. Perez-Garcia, Phys. Rev. E 54, 1560 (1996); Y. N. Young and H. Riecke, Physica D 163, 166 (2002).

[14] H. Rotstein and B. A. Malomed, Physica Scripta 62, 164 (2000).

[15] B. A. Malomed, Physica Scripta 57, 115 (1997); H. Sakaguchi and B. A. Malomed, Physica D 118, 250 (1998).

[16] S. Wabnitz, IEEE Phot. Tech. Lett. 21, 875 (2009).

[17] P. G. Kevrekidis, B. A. Malomed, D. J. Frantzeskakis, and A. R. Bishop, Phys. Rev. E 67, 036614 (2003); Z. Rapti, A. Trombettoni, P. G. Kevrekidis, D. J. Frantzeskakis, and B. A. Malomed, and A. R. Bishop, Phys. Lett. A 330, 95 (2004).

[18] A. Mering and M. Fleischhauer, Phys. Rev. A 81, 011603(R) (2010).

[19] G. Gligorić, A. Maluckov, M. Stepić, L. Hadžievski, and B. A. Malomed, Phys. Rev. A 82, 033624 (2010).

[20] W. Zhang, D. L. Zhou, M.-S. Chang, M. S. Chapman, and L. You, Phys. Rev. Lett. 95, 180403 (2005); M. Uchiyama, J. Ieda, and M. Wadati, J. Phys. Soc. Jpn. 75, 064002 (2006); H. Saito, Y. Kawaguchi, and M. Ueda, Phys. Rev. A 75, 013621 (2007); H. E. Nistazakis, D. J. Frantzeskakis, P. G. Kevrekidis, B. A. Malomed, R. Carretero-González, and A. R. Bishop, ibid. A 76, 063603 (2007); Z.-D. Li, Q.-Y. Li, P.-B. He, J.-Q. Liang, W. M. Liu, and G. Fu, Phys. Rev. A 81, $015602(2010)$.

[21] V. Steinberg, G. Ahlers, and D. S. Cannell, Physica Scripta 32, 534 (1985); Y. C. Hu, R. Ecke, and G. Ahlers, Phys. Rev. E 48, 4399 (1993).

[22] Ye. Larionova, U. Peschel, A. Esteban-Martin, J. Garcia Monreal, and C. O. Weiss, Phys. Rev. A 69, 033803 (2004).

[23] S. Pitois, G. Millot, and S. Wabnitz, Phys. Rev. Lett. 81, 1409 (1998).

[24] H. Zhang, D. Y. Tang, L. M. Zhao, and X. Wu, Phys. Rev. B 80, 052302 (2009); H. Zhang, D. Y. Tang, and L. M. Zhao, Opt. Exp. 18, 4428 (2010).

[25] L. E. Sadler, J. M. Higbie, S. R. Leslie, M. Vengalattor, and D. M. Stamper-Kurn, Nature 443, 312 (2006); N. Gemelke, X. Zhang, C.-L. Hung, and C. Chin, ibid. 460, 995 (2009).

[26] D. T. Son and M. A. Stephanov, Phys. Rev. A 65, 063621 (2002); B. Deconinck, P. G. Kevrekidis, H. E. Nistazakis, and D. J. Frantzeskakis, ibid. A 70, 063605 (2004); M. I. Merhasin, B. A. Malomed, and R. Driben, J. Phys. B: At. Mol. Opt. 38, 877 (2005); A. Niederberger, B. A. Malomed, and M. Lewenstein, Phys. Rev. A 82, 043622 (2010). 
[27] R. S. Tasgal and B. A. Malomed, Physica Scripta 60, 418 (1999).

[28] B. A. Malomed and A. A. Nepomnyashchy, Europhys. Lett. 27, 649 (1994); B. A. Malomed, Phys. Rev. E 58, 7928 (1998).

[29] I. V. Barashenkov and E. Y. Panova, Physica D 69, 114 (1993); I. V. Barashenkov, Phys. Rev. Lett. 77, 1193 (1996).

[30] J. F. Dillon, J. P. Remeika, and C. R. Staton, J. Appl. Phys. 41, 4613 (1970); Y. Linzon, K. A. Rutkowska, B. A. Malomed, and R. Morandotti, Phys. Rev. Lett. 103, 053902 (2009); K. A. Rutkowska, B. A. Malomed, and R. Morandotti, Opt. Exp. 18, 8879 (2010).

[31] R. W. Boyd, Nonlinear Optics (Academic Press, San Diego: 2008).

[32] P. Coullet, L. Gil, and F. Rocca, Opt. Commun. 73, 403 (1989); G. A. Swartzlander and C. T. Law, Phys. Rev. Lett. 69, 2503 (1992); I. V. Basistiy, V. Y. Bazhenov, M. S. Soskin, and M. V. Vasnetsov, Opt. Commun. 103, 422 (1993); D. Rozas, C. T. Law, and G. A. Swartzlander, J. Opt. Soc. Am. B 14, 3054 (1997); M. S. Soskin, V. N. Gorshkov, M. V. Vasnetsov, J. T. Malos, and N. R. Heckenberg, Phys. Rev. A 56, 4064 (1997); D. M. Palacios, I. D. Maleev, A. S. Marathay, and G. A. Swartzlander, Phys. Rev. Lett. 92, 143905 (2004).

[33] S. A. Langer, R. E. Goldstein, and D. P. Jackson, Phys. Rev. A 46, 4894 (1992); K. O. Ng and D. Vanderbilt, Phys. Rev. B 52, 2177 (1995); R. P. Cowburn, D. K. Koltsov, A. O. Adeyeye, M. E. Welland, and D. M. Tricker, Phys. Rev. Lett. 83, 1042 (1999).

[34] B. A. Malomed, D. Mihalache, F. Wise, and L. Torner,. J. Optics B: Quant. Semicl. Opt. 7, R53 (2005).

[35] I. L. Bogolyubskii and V. G. Makhankov, JETP Lett. 25, 107 (1977); P. L. Christiansen, P. S. Lomdahl, and N. J. Zabusky, Appl. Phys. Lett. 39, 170 (1981); E. M. Maslov, Phys. Lett. A 151, 47 (1990); A. G. Bratsos, J. Comp. Appl. Math. 206, 251 (2007).

[36] J. Geicke, Physica Scripta 29, 431 (1984).

[37] P. L. Christiansen, N. Grønbech-Jensen, P. S. Lomdahl, and B. A. Malomed, Physica Scripta 55, 131 (1997). 